\title{
Singly heavy baryons with chiral partner structure in a three-flavor chiral model
}

\author{
Yohei Kawakami* and Masayasu Harada $^{\dagger}$ \\ Department of Physics, Nagoya University, Nagoya, 464-8602, Japan
}

(Received 26 February 2019; published 14 May 2019)

\begin{abstract}
We construct an effective hadronic model including singly heavy baryons (SHBs) belonging to the $(\mathbf{3}, \mathbf{3})$ representation under $\mathrm{SU}(3)_{L} \times \mathrm{SU}(3)_{R}$ symmetry, respecting the chiral symmetry and heavy-quark spinflavor symmetry. When the chiral symmetry is spontaneously broken, the SHBs are divided into the baryons with negative parity of $\overline{\mathbf{3}}$ representation under SU(3) flavor symmetry, which are the chiral partners to the ones with positive parity of $\mathbf{6}$ representation. We determine the model parameters from the available experimental data for the masses and strong decay widths of $\Sigma_{c}^{(*)}, \Lambda_{c}(2595), \Xi_{c}(2790)$, and $\Xi_{c}(2815)$. Then, we predict the masses and strong decay widths of other baryons including $\Xi_{b}$ with negative parity. We also study radiative decays of SHBs including $\Omega_{c}^{*}$ and $\Omega_{b}^{*}$ with positive parity.
\end{abstract}

DOI: 10.1103/PhysRevD.99.094016

\section{INTRODUCTION}

The spontaneous chiral symmetry breaking, which is one of the most essential properties of QCD, is expected to generate a part of hadron masses and causes the mass difference between chiral partners. An investigation of chiral partner structure will provide some clues to understand the chiral symmetry. In particular, a study of the chiral partner structure of hadrons, including heavy quarks, gives information which is not obtained from the hadrons including only light quarks.

There are several studies of hadrons including heavy quarks based on the chiral partner structure. The chiral partner structure of heavy-light mesons is studied in, e.g., Refs. [1-5], that of doubly heavy baryons is in, e.g., Refs. [6-8], and the singly heavy baryons (SHBs) are studied in, e.g., Refs. [9-13].

In Ref. [12], we proposed a new chiral partner structure for SHBs including a heavy quark and two light quarks. There, we considered the chiral partners of $\Sigma_{Q}(Q=c, b)$ baryons with positive parity as $\Lambda_{Q}$ baryons with negative parity: a heavy-quark doublet of $\left(\Lambda_{c}\left(2595 ; J^{P}=1 / 2^{-}\right)\right.$, $\left.\Lambda_{c}\left(2625 ; 3 / 2^{-}\right)\right)$is regarded as the chiral partners to the doublet of $\left(\Sigma_{c}\left(2455 ; 1 / 2^{+}\right), \Sigma_{c}\left(2520 ; 3 / 2^{+}\right)\right)$, and $\left(\Lambda_{b}\left(5912 ; 1 / 2^{-}\right), \quad \Lambda_{b}\left(5920 ; 3 / 2^{-}\right)\right)$to $\left(\Sigma_{b}\left(1 / 2^{+}\right)\right.$, $\left.\Sigma_{b}^{*}\left(3 / 2^{+}\right)\right)$. Based on this structure, we predicted the pionic

\footnotetext{
*kawakami@hken.phys.nagoya-u.ac.jp

harada@hken.phys.nagoya-u.ac.jp
}

Published by the American Physical Society under the terms of the Creative Commons Attribution 4.0 International license. Further distribution of this work must maintain attribution to the author(s) and the published article's title, journal citation, and DOI. Funded by SCOAP ${ }^{3}$. and photonic decay widths of these excited SHBs. The results show that, although the decay of $\Lambda_{c}(2595)$ is dominated by the resonant contribution through $\Sigma_{c}(2455)$, nonresonant contributions are important for $\Lambda_{c}(2625), \Lambda_{b}(5912)$, and $\Lambda_{b}(5920)$, which reflects the chiral partner structure. In Ref. [13], its experimental verification was proposed.

In the present work, we extend the chiral partner model in Ref. [12], which is based on $\mathrm{SU}(2)_{L} \times \mathrm{SU}(2)_{R}$ symmetry, to $\mathrm{SU}(3)_{L} \times \mathrm{SU}(3)_{R}$ symmetry. We consider that the SHBs with negative parity which belongs to $\overline{\mathbf{3}}$ representation under $\mathrm{SU}(3)$ flavor symmetry are the chiral partner to the SHBs with positive parity to $\mathbf{6}$ representation. We introduce a chiral field belonging to $(\mathbf{3}, \mathbf{3})$ representation under $\mathrm{SU}(3)_{L} \times \mathrm{SU}(3)_{R}$ symmetry to construct an effective Lagrangian including the interactions to light pseudoscalar mesons. Determining the model parameters from existing experimental data, we give predictions on the masses and pionic decay widths which are not experimentally determined. We also study radiative decays by introducing the interactions with a photon field in a chiral invariant way. The singly heavy baryons have been studied experimentally (e.g., Refs. [14-16]), and theoretically based on chiral models (e.g., Refs. [17-19]), quark models (e.g., Refs. [2026]), the sum rule (e.g., Refs. [27-29]), the Regge theory (e.g., Ref. [30]), lattice simulations (e.g., Refs. [31,32]), and molecule models (e.g., Refs. [33,34]) (See for a review, e.g., Ref. [35] and references therein.). In this paper, we make comparisons of our predictions with those in chiral effective models [17,18], quark models [20,25,30], and lattice simulations [31,32].

This paper is organized as follows: We construct an effective Lagrangian in Sec. II. Sections III and IV are devoted to study the masses and the hadronic decays of 
SHBs. We also study the radiative decays of SHBs in Sec. V. Finally, we give a summary and discussions in Sec. VI.

\section{EFFECTIVE LAGRANGIAN}

In this section, we construct an effective model of SHBs by extending the two-flavor model provided in the previous work [12], to the three-flavor case.

We introduce a set of fields, $S_{Q}^{\mu}(Q=b, c)$, for SHBs in which the light-quark cloud carries the spin 1 and belongs to $(\mathbf{3}, \mathbf{3})$ representation under $\mathrm{SU}(3)_{L} \times \mathrm{SU}(3)_{R}$ symmetry. The field transforms as

$$
S_{Q}^{\mu} \stackrel{\text { Ch. }}{\longrightarrow} g_{R} S_{Q}^{\mu} g_{L}^{T}, \quad(Q=c, b),
$$

where $g_{L, R} \in \mathrm{SU}(3)_{L, R}$. When the chiral symmetry is spontaneously broken, $S_{Q}^{\mu}$ is divided into two parts. One is for the positive parity SHBs belonging to 6 representation under $\mathrm{SU}(3)_{\text {flavor }}$ symmetry, $\hat{B}_{Q}^{6 \mu}$, and another for the negative parity SHBs to $\overline{\mathbf{3}}, \hat{B}_{Q}^{\overline{3} \mu}$ :

$$
S_{Q}^{\mu}=\hat{B}_{Q}^{6 \mu}+\hat{B}_{Q}^{\overline{3} \mu} .
$$

We would like to stress that $\hat{B}_{Q}^{6 \mu}$ and $\hat{B}_{Q}^{\overline{3} \mu}$ are chiral partners to each other in the present model. The physical states are embedded as

$$
\begin{gathered}
\hat{B}_{Q}^{6 \mu}=\left(\begin{array}{ccc}
\Sigma_{Q}^{I=1 \mu} & \frac{1}{\sqrt{2}} \Sigma_{Q}^{I=0 \mu} & \frac{1}{\sqrt{2}} \Xi_{Q}^{I=\frac{1}{2} \mu} \\
\frac{1}{\sqrt{2}} \Sigma_{Q}^{I=0 \mu} & \Sigma_{Q}^{I=-1 \mu} & \frac{1}{\sqrt{2}} \Xi_{Q}^{I=-\frac{1}{2} \mu} \\
\frac{1}{\sqrt{2}} \Xi_{Q}^{I I=\frac{1}{2} \mu} & \frac{1}{\sqrt{2}} \Xi_{Q}^{I=-\frac{1}{2} \mu} & \Omega_{Q}^{\mu}
\end{array}\right), \\
\hat{B}_{Q}^{\overline{3} \mu}=\frac{1}{\sqrt{2}}\left(\begin{array}{ccc}
0 & \Lambda_{Q 1}^{\mu} & \Xi_{Q 1}^{I=\frac{1}{2} \mu} \\
-\Lambda_{Q 1}^{\mu} & 0 & \Xi_{Q 1}^{I=-\frac{1}{2} \mu} \\
-\Xi_{Q 1}^{I=\frac{1}{2} \mu} & -\Xi_{Q 1}^{I=-\frac{1}{2} \mu} & 0
\end{array}\right) .
\end{gathered}
$$

These $B_{Q}^{6 \mu}$ and $B_{Q}^{\overline{3} \mu}$ are decomposed into spin- $3 / 2$ baryon fields and spin- $1 / 2$ fields as

$$
\begin{aligned}
& B_{Q}^{6 \mu}=B_{Q}^{6 * \mu}-\frac{1}{\sqrt{3}}\left(\gamma^{\mu}+v^{\mu}\right) \gamma_{5} B_{Q}^{6}, \\
& B_{Q}^{\overline{3} \mu}=B_{Q}^{\overline{3} * \mu}-\frac{1}{\sqrt{3}}\left(\gamma^{\mu}+v^{\mu}\right) \gamma_{5} B_{Q}^{\overline{3}},
\end{aligned}
$$

where $B_{Q}^{6 * \mu}$ and $B_{Q}^{\overline{3} * \mu}$ denote the spin-3/2 baryon fields, and $B_{Q}^{6}$ and $B_{Q}^{\overline{3}}$ the spin-1/2 fields, respectively. We note that the parity transformation of the $S_{Q}^{\mu}$ field is given by

$$
S_{Q}^{\mu} \stackrel{\mathrm{P}}{\longrightarrow}-\gamma^{0} S_{Q \mu}^{T},
$$

where ${ }^{T}$ denotes the transposition of the $3 \times 3$ matrix in the light-quark flavor space, and that the Dirac conjugate is defined as

$$
\bar{S}_{Q}^{\mu}=S_{Q}^{\mu \dagger} \gamma^{0}
$$

We introduce a $3 \times 3$ matrix field $M$ for scalar and pseudoscalar mesons made from a light quark and a light antiquark, which belongs to the $(\mathbf{3}, \overline{\mathbf{3}})$ representation under the chiral $\mathrm{SU}(3)_{L} \times \mathrm{SU}(3)_{R}$ symmetry. The transformation properties of $M$ under the chiral symmetry and the parity are given by

$$
\begin{gathered}
M \stackrel{\text { Ch. }}{\longrightarrow} g_{L} M g_{R}^{\dagger}, \\
M \stackrel{\mathrm{P}}{\longrightarrow} M^{\dagger} .
\end{gathered}
$$

We assume that the potential terms for $M$ in the model are constructed in such a way that the $M$ has a vacuum expectation value which breaks the chiral symmetry spontaneously:

$$
\langle M\rangle=\left(\begin{array}{ccc}
f_{\pi} & 0 & 0 \\
0 & f_{\pi} & 0 \\
0 & 0 & \sigma_{s}
\end{array}\right),
$$

where $f_{\pi}$ is the pion decay constant and $\sigma_{s}$ is written as $\sigma_{s}=2 f_{K}-f_{\pi}$ with the Kaon decay constant $f_{K}{ }^{1}$. In the following, for studying the decays of the SHBs with emitting pions, we parametrize the field $M$ as

$$
M=\xi\left(\begin{array}{ccc}
f_{\pi} & 0 & 0 \\
0 & f_{\pi} & 0 \\
0 & 0 & \sigma_{s}
\end{array}\right) \xi
$$

where

$$
\xi=e^{i \pi / f_{\pi}},
$$

with $\pi$ being the $3 \times 3$ matrix field including pions as

$$
\pi=\frac{1}{2}\left(\begin{array}{ccc}
\pi^{0} & \sqrt{2} \pi^{+} & 0 \\
\sqrt{2} \pi^{-} & -\pi^{0} & 0 \\
0 & 0 & 0
\end{array}\right) .
$$

In addition, we introduce two fields, one belonging to $(\overline{\mathbf{3}}, \mathbf{1})$ representation under $\mathrm{SU}(3)_{L} \times \mathrm{SU}(3)_{R}$ symmetry and another to $(\mathbf{1}, \overline{\mathbf{3}})$ representation. It is convenient to use antisymmetric $3 \times 3$ matrix fields which transform as

$$
S_{Q L L} \stackrel{\text { Ch. }}{\longrightarrow} g_{L} S_{Q L L} g_{L}^{T}, \quad S_{Q R R} \stackrel{\text { Ch. }}{\longrightarrow} g_{R} S_{Q R R} g_{R}^{T},
$$

\footnotetext{
${ }^{1}$ Here we adopt the normalization of $f_{\pi}=92.4 \mathrm{MeV}$ and $f_{K}=1.197 f_{\pi}$.
} 
where $S_{Q L L}$ and $S_{Q R R}$ denote the fields of $(\overline{\mathbf{3}}, \mathbf{1})$ and $(\mathbf{1}, \overline{\mathbf{3}})$ representations, respectively. They are related to each of the others by parity transformation as

$$
S_{Q L L} \stackrel{\mathrm{P}}{\longrightarrow}-\gamma^{0} S_{Q R R}
$$

We introduce the parity eigenstates as

$$
S_{Q L L}=\hat{A}_{Q}^{\overline{3}}-\hat{C}_{Q}^{\overline{3}}, \quad S_{Q R R}=\hat{A}_{Q}^{\overline{3}}+\hat{C}_{Q}^{\overline{3}},
$$

where $A_{Q}^{\overline{3}}$ and $C_{Q}^{\overline{3}}$ carry the negative and positive parities, respectively. They include the flavor antisymmetric fields as

$$
\hat{A}_{Q}^{\overline{3}}=\left(\begin{array}{ccc}
0 & \Lambda_{Q 2}^{\mu} & \Xi_{Q 2}^{I=\frac{1}{2} \mu} \\
-\Lambda_{Q 2}^{\mu} & 0 & \Xi_{Q 2}^{I=-\frac{1}{2} \mu} \\
-\Xi_{Q 2}^{I=\frac{1}{2} \mu} & -\Xi_{Q 2}^{I=-\frac{1}{2} \mu} & 0
\end{array}\right),
$$

$$
\hat{C}_{Q}^{\overline{3}}=\left(\begin{array}{ccc}
0 & \Lambda_{Q}^{\mu} & \Xi_{Q}^{I=\frac{1}{2} \mu} \\
-\Lambda_{Q}^{\mu} & 0 & \Xi_{Q}^{I=-\frac{1}{2} \mu} \\
-\Xi_{Q}^{I=\frac{1}{2} \mu} & -\Xi_{Q}^{I=-\frac{1}{2} \mu} & 0
\end{array}\right)
$$

These $A_{Q}^{\overline{3}}$ and $C_{Q}^{\overline{3}}$ express spin- $1 / 2$ fields, respectively. Since the particles which are expressed by $A_{Q}^{\overline{3}}$ are still undiscovered, we neglect $A_{Q}^{\overline{3}}$ in the following discussion.

Now, let us write down an effective Lagrangian including the baryon fields $S_{Q}^{\mu}, S_{Q L L}$, and $S_{Q R R}$ together with the meson field $M$, based on the heavy-quark spin-flavor symmetry and the chiral symmetry. We do not consider the terms including more than the square of the $M$ field or more than two derivatives. A possible Lagrangian is given by

$$
\begin{aligned}
\mathcal{L}_{Q}= & -\operatorname{tr} \bar{S}_{Q}^{\mu}(v \cdot i D-\Delta) S_{Q \mu}+\bar{S}_{Q L L}(v \cdot i D) S_{Q L L}+\bar{S}_{Q R R}(v \cdot i D) S_{Q R R}+\frac{g_{1}}{2 f_{\pi}} \operatorname{tr}\left(\bar{S}_{Q}^{\mu} M^{\dagger} M S_{Q \mu}+\bar{S}_{Q \mu}^{T} M M^{\dagger} S_{Q}^{\mu T}\right) \\
& -\frac{g_{2}}{2 f_{\pi}} \operatorname{tr} \bar{S}_{Q}^{\mu} M^{\dagger} S_{Q \mu}^{T} M^{T}-\frac{g_{2}^{v}}{2 m_{\Lambda_{Q}}} \operatorname{tr} \bar{S}_{Q^{\mu}}^{\mu} M^{\dagger} S_{Q \mu}^{T} M^{T}+\frac{\kappa_{1}}{4 f_{\pi}} \operatorname{tr}\left(\bar{S}_{Q}^{\mu} \mathcal{M}^{\dagger} M S_{Q \mu}+\bar{S}_{Q^{\mu}}^{\mu} M^{\dagger} \mathcal{M} S_{Q \mu}+\bar{S}_{Q \mu}^{T} \mathcal{M} M^{\dagger} S_{Q}^{\mu T}+\bar{S}_{Q \mu}^{T} M \mathcal{M}^{\dagger} S_{Q}^{\mu T}\right) \\
& -\frac{\kappa_{2}}{2 f_{\pi}} \operatorname{tr}\left(\bar{S}_{Q}^{\mu} \mathcal{M}^{\dagger} S_{Q \mu}^{T} M^{T}+\bar{S}_{Q}^{\mu} M^{\dagger} S_{Q \mu}^{T} \mathcal{M}^{T}\right)-i \frac{h_{1}^{I}-i h_{1}^{R}}{4 f_{\pi}^{2}} \operatorname{tr}\left(\bar{S}_{Q}^{\mu} M^{\dagger} v \cdot \partial M S_{Q \mu}+\bar{S}_{Q}^{\mu T} M v \cdot \partial M^{\dagger} S_{Q \mu}^{T}\right) \\
& -i \frac{-h_{1}^{I}-i h_{1}^{R}}{4 f_{\pi}^{2}} \operatorname{tr}\left(\bar{S}_{Q}^{\mu} v \cdot \partial M^{\dagger} M S_{Q \mu}+\bar{S}_{Q}^{\mu T} v \cdot \partial M M^{\dagger} S_{Q \mu}^{T}\right)+\frac{h_{2}}{2 f_{\pi}^{2}} \operatorname{tr}\left(\bar{S}_{Q}^{\mu} v \cdot \partial M^{\dagger} S_{Q \mu}^{T} M^{T}+\bar{S}_{Q}^{\mu T} v \cdot \partial M S_{Q \mu} M^{*}\right) \\
& -\frac{g_{3}}{2 f_{\pi}} \operatorname{tr}\left(\bar{S}_{Q L L} \partial^{\mu} M S_{Q \mu}+\bar{S}_{Q}^{\mu} \partial_{\mu} M^{\dagger} S_{Q L L}+\bar{S}_{Q R R} \partial^{\mu} M^{\dagger} S_{Q \mu}^{T}+\bar{S}_{Q}^{\mu T} \partial_{\mu} M S_{Q R R}\right)
\end{aligned}
$$

where $m_{\Lambda_{Q}}(Q=c, b)$ are the masses of $\Lambda_{c}(2286)$ and $\Lambda_{b}$ in the ground state, and $\Delta$ provides the difference between the chiral invariant mass of $S_{Q}^{\mu}$ and that of $S_{Q L L}$ and $S_{Q R R}$. $g_{i}(i=1,2,3), g_{2}^{v}, \kappa_{i}(i=1,2), h_{1}^{I}, h_{1}^{R}$, and $h_{2}$ are dimensionless coupling constants. We note that we include the $g_{2}^{v}$-term to incorporate the heavy-flavor violation needed for explaining the mass differences of charm and bottom sectors (See Ref. [12].). Although we can add heavy-quark flavor violation terms corresponding to the $g_{1}$ term, such contributions are absorbed into the definition of $\Delta$. We expect that heavy-quark flavor violating contributions to terms other than the $g_{2}^{v}$-term are small. Since thresholds of $B_{Q}^{6 *} \rightarrow B_{Q}^{6} \pi$ are not open, the related terms are not included here. We note that the above chiral partner may not be necessarily a three-quark state but can be also a molecular state such as the one in Ref. [34].

\section{MASSES AND ONE-PION DECAYS}

In this section, we determine the coupling constants $g_{2}$, $g_{2}^{v}$, and $\kappa_{2}$ from masses of relevant SHBs, and $g_{3}$ from
$\Sigma_{c}^{(*)} \rightarrow \Lambda_{c} \pi$ decays. Then we make predictions of the onepion decay widths of other members of the flavor 6 representation.

When the chiral symmetry is spontaneously broken, the light meson field $M$ acquires its vacuum expectation value as in Eq. (9). Then the masses of the particles included in the model are expressed as

$$
\begin{gathered}
M\left(\Sigma_{Q}\right)=M_{\Lambda_{Q}}+\Delta+g_{1} f_{\pi}-\frac{g_{2}^{Q}}{2} f_{\pi}+\bar{\kappa}_{1}-\bar{\kappa}_{2} \\
M\left(\Xi_{Q}^{\prime}\right)=M_{\Lambda_{Q}}+\Delta+g_{1} \frac{f_{\pi}^{2}+\sigma_{s}^{2}}{2 f_{\pi}}-\frac{g_{2}^{Q}}{2} \sigma_{s}+\bar{\kappa}_{1} \frac{f_{\pi}+\sigma_{s} \frac{m_{s}}{\bar{m}}}{2 f_{\pi}} \\
-\bar{\kappa}_{2} \frac{f_{\pi} \frac{m_{s}}{\bar{m}}+\sigma_{s}}{2 f_{\pi}} \\
M\left(\Omega_{Q}\right)=M_{\Lambda_{Q}}+\Delta+g_{1} \frac{\sigma_{s}^{2}}{f_{\pi}}-\frac{g_{2}^{Q}}{2} \frac{\sigma_{s}^{2}}{f_{\pi}}+\bar{\kappa}_{1} \frac{m_{s}}{\bar{m}} \frac{\sigma_{s}}{f_{\pi}}-\bar{\kappa}_{2} \frac{m_{s}}{\bar{m}} \frac{\sigma_{s}}{f_{\pi}}
\end{gathered}
$$




$$
\begin{aligned}
M\left(\Lambda_{Q 1}\right)= & M_{\Lambda_{Q}}+\Delta+g_{1} f_{\pi}+\frac{g_{2}^{Q}}{2} f_{\pi}+\bar{\kappa}_{1}+\bar{\kappa}_{2} \\
M\left(\Xi_{Q 1}\right)= & M_{\Lambda_{Q}}+\Delta+g_{1} \frac{f_{\pi}^{2}+\sigma_{s}^{2}}{2 f_{\pi}}+\frac{g_{2}^{Q}}{2} \sigma_{s} \\
& +\bar{\kappa}_{1} \frac{f_{\pi}+\sigma_{s} \frac{m_{s}}{2 f_{\pi}}}{2 f_{\pi}}+\bar{\kappa}_{2} \frac{f_{\pi} \frac{m_{s}}{\bar{m}}+\sigma_{s}}{2 f_{\pi}},
\end{aligned}
$$

where $\bar{\kappa}_{i}=\kappa_{i} \bar{m}$, and $g_{2}^{Q}$ is defined as

$$
g_{2}^{Q}=g_{2}+g_{2}^{v} \frac{f_{\pi}}{m_{\Lambda_{Q}}}
$$

We determine the fraction of strange quark mass $m_{s}$ and up or down quark mass $\bar{m}$ from the masses of the pion and kaon as $m_{s} / \bar{m}=25.9$ using

$$
\frac{m_{K}^{2}}{m_{\pi}^{2}}=\frac{m_{s}+\bar{m}}{2 \bar{m}}
$$

In the present analysis, we assign the following physical states to the flavor $\overline{\mathbf{3}}$ representation:

$$
\begin{gathered}
\left(\Lambda_{c 1}, \Lambda_{c 1}^{*}\right)=\left(\Lambda_{c}\left(2595 ; J^{P}=1 / 2^{-}\right), \Lambda_{c}\left(2625 ; 3 / 2^{-}\right)\right), \\
\left(\Xi_{c 1}, \Xi_{c 1}^{*}\right)=\left(\Xi_{c}\left(2790 ; J^{P}=1 / 2^{-}\right), \Lambda_{c}\left(2815 ; 3 / 2^{-}\right)\right)
\end{gathered}
$$

and they are the chiral partner to the flavor 6 representation:

$$
\begin{aligned}
\left(\Sigma_{c}, \Sigma_{c}^{*}\right) & =\left(\Sigma_{c}\left(2455 ; 1 / 2^{+}\right), \Sigma_{c}\left(2520 ; 3 / 2^{+}\right)\right), \\
\left(\Xi_{c}^{\prime}, \Xi_{c}^{\prime *}\right) & =\left(\Xi_{c}^{\prime}\left(1 / 2^{+}\right), \Xi_{c}^{\prime}\left(3 / 2^{+}\right)\right), \\
\left(\Omega_{c}, \Omega_{c}^{*}\right) & =\left(\Omega_{c}\left(1 / 2^{+}\right), \Omega_{c}\left(2770 ; 3 / 2^{+}\right)\right) .
\end{aligned}
$$

In the bottom sector, $\overline{\mathbf{3}}$ includes

$$
\begin{aligned}
& \left(\Lambda_{b 1}, \Lambda_{b 1}^{*}\right)=\left(\Lambda_{c}\left(5912 ; J^{P}=1 / 2^{-}\right), \Lambda_{c}\left(5920 ; 3 / 2^{-}\right)\right), \\
& \left(\Xi_{b 1}, \Xi_{b 1}^{*}\right)=\left(\Xi_{b}\left(1 / 2^{-}\right), \Xi_{b}\left(3 / 2^{-}\right)\right),
\end{aligned}
$$

and 6 includes

$$
\begin{aligned}
& \left(\Sigma_{b}, \Sigma_{b}^{*}\right)=\left(\Sigma_{b}\left(1 / 2^{+}\right), \Sigma_{b}\left(3 / 2^{+}\right)\right), \\
& \left(\Xi_{b}^{\prime}, \Xi_{b}^{* *}\right)=\left(\Xi_{b}^{\prime}\left(5935 ; 1 / 2^{+}\right), \Xi_{c}\left(5945,5955 ; 3 / 2^{+}\right)\right), \\
& \left(\Omega_{b}, \Omega_{b}^{*}\right)=\left(\Omega_{b}\left(1 / 2^{+}\right), \Omega_{b}\left(3 / 2^{+}\right)\right) .
\end{aligned}
$$

We list experimental data of their masses and full decay widths [36] in Table I.

Here, we cannot determine the values of $\Delta, g_{1}$, and $\kappa_{1}$, separately. Instead, we introduce
TABLE I. Experimental data of masses and decay widths of heavy baryons included in the present analysis.

\begin{tabular}{lccc}
\hline \hline Particle & $J^{P}$ & Mass $(\mathrm{MeV})$ & Full width $(\mathrm{MeV})$ \\
\hline$\Lambda_{c}$ & $1 / 2^{+}$ & $2286.46 \pm 0.14$ & No strong decays \\
$\Xi_{c}^{+}$ & $1 / 2^{+}$ & $2467.87 \pm 0.30$ & No strong decays \\
$\Xi_{c}^{0}$ & $1 / 2^{+}$ & $2470.87_{-0.31}^{+0.28}$ & No strong decays \\
$\Sigma_{c}^{++}(2455)$ & $1 / 2^{+}$ & $2453.97 \pm 0.14$ & $1.89_{-0.18}^{+0.09}$ \\
$\Sigma_{c}^{+}(2455)$ & $1 / 2^{+}$ & $2452.9 \pm 0.4$ & $<4.6$ \\
$\Sigma_{c}^{0}(2455)$ & $1 / 2^{+}$ & $2453.75 \pm 0.14$ & $1.83_{-0.19}^{+0.11}$ \\
$\Sigma_{c}^{++}(2520)$ & $3 / 2^{+}$ & $2518.41_{-0.19}^{+0.21}$ & $14.78_{-0.40}^{+0.30}$ \\
$\Sigma_{c}^{+}(2520)$ & $3 / 2^{+}$ & $2517.5 \pm 1.3$ & $<17$ \\
$\Sigma_{c}^{0}(2520)$ & $3 / 2^{+}$ & $2518.48 \pm 0.20$ & $15.3_{-0.5}^{+0.4}$
\end{tabular}

$\Xi_{c}^{\prime+} \quad 1 / 2^{+} \quad 2577.4 \pm 1.2$

$\begin{array}{lll}\Xi_{c}^{\prime 0} & 1 / 2^{+} & 2578.8 \pm 0.5\end{array}$

$\Xi_{c}^{+}(2645) \quad 3 / 2^{+}$

$2645.53 \pm 0.31$

$\Xi_{c}^{0}(2645) \quad 3 / 2^{+}$

$2646.32 \pm 0.31$

$\Omega_{c}$

$2695.2 \pm 1.7$

$\Omega_{c}(2770) \quad 3 / 2^{+}$

$2765.9 \pm 2.0$

$\Lambda_{c}(2595) \quad 1 / 2^{-}$

$2595.25 \pm 0.28$

$\Lambda_{c}(2625) \quad 3 / 2^{-}$

$2628.11 \pm 0.19$

$\Xi_{c}^{+}(2790) \quad 1 / 2^{-}$

$\Xi_{c}^{0}(2790) \quad 1 / 2^{-}$

$\Xi_{c}^{+}(2815) \quad 3 / 2^{-}$

$\Xi_{c}^{0}(2815) \quad 3 / 2^{-}$

$2792.0 \pm 0.5$

$2792.8 \pm 1.2$

$2816.67 \pm 0.31$

$2820.22 \pm 0.32$

No strong decays No strong decays

$2.14 \pm 0.19$

$2.35 \pm 0.18 \pm 0.13$

No strong decays

No strong decays
$2.59 \pm 0.30 \pm 0.47$ $<0.97$

$8.9 \pm 0.6 \pm 0.8$

$10.0 \pm 0.7 \pm 0.8$

$2.43 \pm 0.20 \pm 0.17$

$2.54 \pm 0.18 \pm 0.14$

$\Lambda_{b} \quad 1 / 2^{+}$

$5619.58 \pm 0.17$

No strong decays

$\begin{array}{lll}\Xi_{b}^{0} & 1 / 2^{+} & 5791.9 \pm 0.5\end{array}$

$\Xi_{b}^{-} \quad 1 / 2^{+} \quad 5794.5 \pm 1.4$

$\Sigma_{b}^{+} \quad 1 / 2^{+}$

$\Sigma_{b}^{0} \quad 1 / 2^{+}$

$\Sigma_{b}^{-} \quad 1 / 2^{+}$

$\Sigma_{b}^{*+} \quad 3 / 2^{+}$

$\Sigma_{b}^{* 0} \quad 3 / 2^{+}$

$5811.3_{-0.8}^{+0.9} \pm 1.7$

$5815.5_{-0.5}^{+0.6} \pm 1.7$

$5832.1 \pm 0.7_{-1.8}^{+1.7}$

$\Sigma_{b}^{*-} \quad 3 / 2^{+}$

$5835.1 \pm 0.6_{-1.8}^{+1.7}$

No strong decays

No strong decays

$\Xi_{b}^{\prime 0} \quad 1 / 2^{+}$

$\Xi_{b}^{\prime-}(5935) \quad 1 / 2^{+}$

$\Xi_{b}^{0}(5945) \quad 3 / 2^{+}$

$\Xi_{b}^{-}(5955) \quad 3 / 2^{+}$

$5935.02 \pm 0.02 \pm 0.05$

$5949.8 \pm 1.4$

$$
\begin{gathered}
9.7_{-2.8}^{+3.8+1.2} \\
\cdots \\
4.9_{-2.1}^{+3.1} \pm 1.1 \\
11.5_{-2.2}^{+2.7}+1.0 \\
\ldots \\
7.5_{-1.8}^{+2.2}+0.9 \\
\ldots
\end{gathered}
$$

$$
<0.08
$$

$0.90 \pm 0.16 \pm 0.08$

$5955.33 \pm 0.12 \pm 0.05 \quad 1.65 \pm 0.31 \pm 0.10$

$\Omega_{b} \quad 1 / 2^{+}$

$\Omega_{b}^{*} \quad 3 / 2^{+}$

$6046.1 \pm 1.7$

$\Lambda_{b}(5912) \quad 1 / 2^{-}$

$\Lambda_{b}(5920) \quad 3 / 2^{-}$

$5912.18 \pm 0.13 \pm 0.17$

$5919.90 \pm 0.19$

$\Xi_{b 1}^{0} \quad 1 / 2^{-}$

$\begin{array}{ll}\Xi_{b 1}^{-} & 1 / 2^{-} \\ \Xi_{b 1}^{0} & 3 / 2^{-} \\ \Xi_{b 1}^{-} & 3 / 2^{-}\end{array}$
$<0.66$

No strong decays

$$
<0.63
$$

$\cdots$

..

..

...

. 


$$
\begin{aligned}
\bar{\Delta} & =\Delta+g_{1} f_{\pi}+\bar{\kappa}_{1} \\
\Delta_{s} & =\Delta+g_{1} \frac{f_{\pi}^{2}+\sigma_{s}^{2}}{2 f_{\pi}}+\bar{\kappa}_{1} \frac{f_{\pi}+\sigma_{s} \frac{m_{s}}{\bar{m}}}{2 f_{\pi}} \\
\Delta_{\Omega} & =\Delta+g_{1} \frac{\sigma_{s}^{2}}{f_{\pi}}+\bar{\kappa}_{1} \frac{m_{s}}{\bar{m}} \frac{\sigma_{s}}{f_{\pi}},
\end{aligned}
$$

to rewrite mass formulas as

$$
\begin{aligned}
& M\left(\Sigma_{Q}\right)=M_{\Lambda_{Q}}+\bar{\Delta}-\frac{g_{2}^{Q}}{2} f_{\pi}-\bar{\kappa}_{2}, \\
& M\left(\Xi_{Q}^{\prime}\right)=M_{\Lambda_{Q}}+\Delta_{s}-\frac{g_{2}^{Q}}{2} \sigma_{s}-\bar{\kappa}_{2} \frac{f_{\pi} \frac{m_{s}}{\bar{m}}+\sigma_{s}}{2 f_{\pi}}, \\
& M\left(\Omega_{Q}\right)=M_{\Lambda_{Q}}+\Delta_{\Omega}-\frac{g_{2}^{Q}}{2} \frac{\sigma_{s}^{2}}{f_{\pi}}-\bar{\kappa}_{2} \frac{m_{s}}{\bar{m}} \frac{\sigma_{s}}{f_{\pi}}, \\
& M\left(\Lambda_{Q 1}\right)=M_{\Lambda_{Q}}+\bar{\Delta}+\frac{g_{2}^{Q}}{2} f_{\pi}+\bar{\kappa}_{2}, \\
& M\left(\Xi_{Q 1}\right)=M_{\Lambda_{Q}}+\Delta_{s}+\frac{g_{2}^{Q}}{2} \sigma_{s}+\bar{\kappa}_{2} \frac{f_{\pi} \frac{m_{s}}{\bar{m}}+\sigma_{s}}{2 f_{\pi}} .
\end{aligned}
$$

We estimate the values of mass parameters and coupling constants in the charm sector from experimental data in a way explained in Ref. [12]: We calculate the spin-averaged mass of SHBs in a heavy-quark multiplet by including errors to include the masses of members belonging to the multiplet as shown in Table II.

To include the heavy-quark flavor-symmetry violation, we determined the value of $g_{2}^{b}$ from the mass difference between spin-averaged masses of $\Lambda_{b 1}^{(*)}$ and $\Sigma_{b}^{(*)}$. In addition, we use the weighted average of $\Sigma_{c}^{++} \rightarrow \Lambda_{c}^{+} \pi^{+}$, $\Sigma_{c}^{0} \rightarrow \Lambda_{c}^{+} \pi^{-}, \Sigma_{c}^{*++} \rightarrow \Lambda_{c}^{+} \pi^{+}$, and $\Sigma_{c}^{* 0} \rightarrow \Lambda_{c}^{+} \pi^{-}$to determine the coupling constant $g_{3}$ as done in Ref. [12]. We show the estimated values of model parameters in Table III.

Using the estimated value of $g_{3}$, we predict the decay widths of $\Sigma_{Q}^{(*)} \rightarrow \Lambda_{Q} \pi$ and $\Xi_{Q}^{\prime(*)} \rightarrow \Xi_{Q} \pi$ as shown in Table IV. These predictions are consistent with

\begin{tabular}{|c|c|c|}
\hline Decay modes & Our model (MeV) & Experimental $(\mathrm{MeV})$ \\
\hline$\Sigma_{c}^{++} \rightarrow \Lambda_{c}^{+} \pi^{+}$ & $1.96_{-014}^{+0.07}$ & $1.89_{-0.18}^{+0.09}$ \\
\hline$\Sigma_{c}^{+} \rightarrow \Lambda_{c}^{+} \pi^{0}$ & $2.28_{-0.17}^{+0.09}$ & $<4.6$ \\
\hline$\Sigma_{c}^{0} \rightarrow \Lambda_{c}^{+} \pi^{-}$ & $1.94_{-0.14}^{+0.07}$ & $1.83_{-0.19}^{+0.11}$ \\
\hline$\Sigma_{c}^{*++} \rightarrow \Lambda_{c}^{+} \pi^{+}$ & $14.7_{-1.1}^{+0.6}$ & $14.78_{-0.40}^{+0.30}$ \\
\hline$\Sigma_{c}^{*+} \rightarrow \Lambda_{c}^{+} \pi^{0}$ & $15.3_{-1.1}^{+0.6}$ & $<17$ \\
\hline$\Sigma_{c}^{* 0} \rightarrow \Lambda_{c}^{-} \pi^{0}$ & $14.7_{-1.1}^{+0.6}$ & $15.3_{-0.5}^{+0.4}$ \\
\hline$\Sigma_{b}^{+} \rightarrow \Lambda_{b}^{0} \pi^{+}$ & $6.14_{-0.45}^{+0.23}$ & $9.7_{-2.8-1.1}^{+3.8+1.2}$ \\
\hline$\Sigma_{b}^{0} \rightarrow \Lambda_{b}^{0} \pi^{0}$ & $7.27_{-0.53}^{+0.27}$ & $\cdots$ \\
\hline$\Sigma_{b}^{-} \rightarrow \Lambda_{b}^{0} \pi^{-}$ & $7.02_{-0.51}^{+0.27}$ & $4.9_{-2.1}^{+3.1} \pm 1.1$ \\
\hline$\Sigma_{b}^{*+} \rightarrow \Lambda_{b}^{0} \pi^{+}$ & $11.0_{-0.8}^{+0.4}$ & $11.5_{-2.2}^{+2.7+1.0}$ \\
\hline$\Sigma_{b}^{* 0} \rightarrow \Lambda_{b}^{0} \pi^{0}$ & $12.3_{-0.9}^{+0.5}$ & $\ldots$ \\
\hline$\Sigma_{b}^{*-} \rightarrow \Lambda_{b}^{0} \pi^{-}$ & $11.9_{-0.9}^{+0.9}$ & $7.5_{-1.8}^{+2.2+0.9}$ \\
\hline$\Xi_{c}^{\prime+} \rightarrow \Xi_{c}^{+} \pi^{0}$ & $\cdots$ & No strong decays \\
\hline$\Xi_{c}^{\prime+} \rightarrow \Xi_{c}^{0} \pi^{+}$ & $\cdots$ & No strong decays \\
\hline$\Xi_{c}^{\prime *+} \rightarrow \Xi_{c} \pi$ & $2.39_{-0.17}^{+0.09}$ & $2.14 \pm 0.19$ \\
\hline$\Xi_{c}^{*+} \rightarrow \Xi_{c}^{+} \pi^{0}$ & $0.953_{-0.069}^{+0.036}$ & $\ldots$ \\
\hline$\Xi_{c}^{*+} \rightarrow \Xi_{c}^{0} \pi^{+}$ & $1.44_{-0.10}^{+0.05}$ & $\cdots$ \\
\hline$\Xi_{c}^{\prime * 0} \rightarrow \Xi_{c} \pi$ & $2.57_{-0.19}^{+0.10}$ & $2.35 \pm 0.18 \pm 0.13$ \\
\hline$\Xi_{c}^{\prime * 0} \rightarrow \Xi_{c}^{0} \pi^{0}$ & $0.873_{-0.063}^{+0.033}$ & $\ldots$ \\
\hline$\Xi_{c}^{\prime * 0} \rightarrow \Xi_{c}^{+} \pi^{-}$ & $1.70_{-0.12}^{+0.06}$ & $\cdots$ \\
\hline$\Xi_{b}^{\prime 0} \rightarrow \Xi_{b} \pi$ & $0.0806_{-0.0059}^{+0.0030}$ & $\cdots$ \\
\hline$\Xi_{b}^{\prime 0} \rightarrow \Xi_{b}^{0} \pi^{0}$ & $0.0746_{-0.0054}^{+0.0028}$ & $\cdots$ \\
\hline$\Xi_{b}^{\prime 0} \rightarrow \Xi_{b}^{-} \pi^{+}$ & $0.00601_{-0.00044}^{+0.00023}$ & $\cdots$ \\
\hline$\Xi_{b}^{\prime-} \rightarrow \Xi_{b} \pi$ & $0.0853_{-0.0062}^{+0.0032}$ & $<0.08$ \\
\hline$\Xi_{b}^{\prime-} \rightarrow \Xi_{b}^{-} \pi^{0}$ & $0.0413_{-0.0030}^{+0.0016}$ & $\cdots$ \\
\hline$\Xi_{b}^{\prime-} \rightarrow \Xi_{b}^{0} \pi^{-}$ & $0.0440_{-0.0032}^{+0.0017}$ & $\cdots$ \\
\hline$\Xi_{b}^{\prime * 0} \rightarrow \Xi_{b} \pi$ & $0.813_{-0.059}^{+0.031}$ & $0.90 \pm 0.16 \pm 0.08$ \\
\hline$\Xi_{b}^{\prime \prime 0} \rightarrow \Xi_{b}^{0} \pi^{0}$ & $0.378_{-0.027}^{+0.014}$ & $\ldots$ \\
\hline$\Xi_{b}^{\prime * 0} \rightarrow \Xi_{b}^{-} \pi^{+}$ & $0.435_{-0.032}^{+0.016}$ & $\cdots$ \\
\hline$\Xi_{b}^{*-} \rightarrow \Xi_{b} \pi$ & $1.30_{-0.09}^{+0.05}$ & $1.65 \pm 0.31 \pm 0.10$ \\
\hline$\Xi_{b}^{\prime *-} \rightarrow \Xi_{b}^{-} \pi^{0}$ & $0.459_{-0.033}^{+0.017}$ & $\ldots$ \\
\hline$\Xi_{b}^{\prime *-} \rightarrow \Xi_{b}^{0} \pi^{-}$ & $0.843_{-0.061}^{+0.032}$ & $\cdots$ \\
\hline
\end{tabular}

TABLE II. Spin-averaged masses and widths used as inputs to determine the model parameters.

\begin{tabular}{lc}
\hline \hline Input & Value $(\mathrm{MeV})$ \\
\hline$M\left(\Lambda_{c}\right)$ & 2286.46 \\
$M\left(\Sigma_{c}^{(*)}\right)$ & $2496.6_{-44.6}^{+21.5}$ \\
$M\left(\Xi_{c}^{(*)}\right)$ & $2623.3_{-45.2}^{+22.6}$ \\
$M\left(\Omega_{c}^{(*)}\right)$ & $2742.3_{-447 .}^{+23.6}$ \\
$M\left(\Lambda_{c 1}^{(*)}\right)$ & $2617.16_{-21.91}^{+10.95}$ \\
$M\left(\Xi_{c 1}^{(*)}\right)$ & $2809.8_{-17.4}^{+8.7}$ \\
$\Gamma\left(\Sigma_{c}^{(*)} \rightarrow \Lambda_{c} \pi\right)$ & $10.6_{-9.0}^{+4.9}$ \\
$M\left(\Lambda_{b}\right)$ & 5619.58 \\
$M\left(\Lambda_{b 1}^{(*)}\right)-M\left(\Sigma_{b}^{(*)}\right)$ & $90.5_{-4.2}^{+8.3}$ \\
\hline \hline
\end{tabular}

TABLE III. Estimated values of model parameters.

\begin{tabular}{lc}
\hline \hline Parameter & Value \\
\hline $\bar{\Delta}$ & $270_{-34}^{+17} \mathrm{MeV}$ \\
$\Delta_{s}$ & $430_{-31}^{+16} \mathrm{MeV}$ \\
$\Delta_{\Omega}$ & $600_{-27}^{+13} \mathrm{MeV}$ \\
$g_{2}^{c}$ & $1.28_{-0.11}^{+0.20}$ \\
$g_{2}^{b}$ & $0.980_{-0.046}^{+0.090}$ \\
$\bar{\kappa}_{2}$ & $0.807_{-0.23}^{+0.47}$ \\
$g_{3}$ & $0.688_{-0.025}^{+0.013}$ \\
\hline \hline
\end{tabular}

TABLE IV. Decay widths of $\Sigma_{Q}^{(*)} \rightarrow \Lambda_{Q} \pi$ and $\Xi_{Q}^{\prime(*)} \rightarrow \Xi_{Q} \pi$ predicted in our model. 
TABLE V. Predicted values of the spin-averaged masses of bottom baryons. For comparison we list the spin averages of experimentally observed masses and the predicted values in Refs. [30,37].

\begin{tabular}{lcccc}
\hline \hline Particle & (Our model) & {$[30]$} & {$[37]$} & Experimental (Spin averaged) \\
\hline$\Sigma_{b}^{(*)}$ & $5843_{-37}^{+20}$ & $5811-5835$ & $\ldots$ & 5826.9 \\
$\Xi_{b}^{\prime(*)}$ & $5975_{-37}^{+18}$ & $\ldots$ & $\ldots$ & 5946.7 \\
$\Omega_{b}^{(*)}$ & $6102_{-36}^{+15}$ & $6048-6086$ & $\ldots$ & $6046.1($ spin-1/2) \\
$\Lambda_{b 1}^{(*)}$ & $5936_{-36}^{+20}$ & $5980-6000$ & $\ldots$ & 5917.33 \\
$\Xi_{b 1}^{(*)}$ & $6124_{-34}^{+20}$ & $6129-6151$ & 6096,6102 & $\ldots$ \\
\hline \hline
\end{tabular}

experimental data because light flavor symmetry violation and heavy-quark symmetry violation are small for the $g_{3}$-term.

We can estimate the masses of bottom baryons included in our model using the parameters in Table III. In the present analysis, we assume heavy-quark spin symmetry, so that we predict the spin-averaged masses which are shown in Table V. Here, we show the results in Refs. [30,37] and experimental values for comparison. We note that, in Table $\mathrm{V}$, we just put the minimum and maximum values predicted for the members in a multiplet in Ref. [30]. This table shows that our predictions are consistent with those in Ref. [30,37].

We can see that our predictions for $\Sigma_{b}^{(*)}, \Xi_{b}^{\prime(*)}$, and $\Lambda_{b 1}^{(*)}$ are consistent with the spin-averaged masses of experimentally observed masses. For $\Omega_{b}$, only the mass of the spin-1/2 member is known experimentally. Although our prediction of the spin average is slightly larger than the observed mass of the spin- $1 / 2$ member, we expect that the spin-3/2 member is slightly heavier which makes the spinaveraged masses larger and consistent with our prediction. Future experimental observations of the spin- $3 / 2$ member as well as $\Xi_{b 1}^{(*)}$ will be a test of the present model. We note that $\Xi_{b 1}^{(*)}$ in the present analysis are unlikely to make a multiplet including $\Xi_{b}(6227)$ reported in Ref. [38], since the predicted mass of $\Xi_{b 1}^{(*)}$ is about $100 \mathrm{MeV}$ smaller than the observed mass of $\Xi_{b}(6227)$.

\section{PION DECAYS OF SINGLE HEAVY BARYONS WITH NEGATIVE PARITY}

In this section, we consider decays of $B_{Q}^{\overline{3}(*)}$, the negative parity excited SHBs belonging to the flavor 3 representation. The main modes of $\Lambda_{Q 1}^{(*)}$ are three-body decay, $\Lambda_{Q 1} \rightarrow$ $\Lambda_{Q} \pi \pi$ because $\Lambda_{Q 1}^{(*)} \rightarrow \Sigma_{Q}^{(*)} \pi$ decay thresholds are closed in most cases. In the decays of $\Xi_{c 1}^{(*)}$, the decay thresholds of $\Xi_{c 1}^{(*)} \rightarrow \Xi_{c}^{(*)} \pi$ are completely open, so the main mode is the two-body decay.

In Ref. [12], we used the two-pion decay width of $\Lambda_{c}(2595)$ to determine the values of derivative coupling constants, $h_{1}^{I}$ and $h_{2}$. Here, we also include the decay widths of $\Xi_{c}(2790)$ and $\Xi_{c}(2815)$. There exists violation of the heavy-quark spin symmetry between the decay widths of $\Xi_{c}(2790)$ and $\Xi_{c}(2815)$. Instead of treating this violation precisely, we include the violation as systematic errors of the model. Therefore, we use values of a decay width of $\Lambda_{c}(2595)$ and a spin-averaged decay width between $\Xi_{c}(2790)$ and $\Xi_{c}(2815)$ as inputs to determine $h_{1}^{I}$ and $h_{2}$. The region colored by dark purple in Fig. 1 shows the allowed values of $h_{1}^{I}$ and $h_{2}$ determined from the decay width of $\Lambda_{c}(2595)$ where the errors of $g_{2}^{c}, g_{3}$ and the total width with $\Lambda(2595)$ are taken into account. The region indicated by light purple is obtained from the spin-averaged width of $\Xi_{c}(2790)$ and $\Xi_{c}(2815)$ with the errors of model parameters included.

In the following analysis, we use the values of $h_{1}^{I}$ and $h_{2}$ in the overlapped region of two colors in Fig. 1 to make predictions of the decay widths of excited SHBs with negative parity. We show the results of total decay widths in Table VI, where we list predictions by the quark model in Refs. [25,37] for comparison.

We note that we use the predicted masses of $\Xi_{b}$ with the negative parity shown in Table $\mathrm{V}$ by including their errors. So, the predicted decay widths take a wide range of values, which include predictions in Refs. [25,37]. In particular, since the minimum value shown in Table $\mathrm{V}$ is very close to the threshold of the relevant decays, the minimum values of the predictions of one-pion decays of

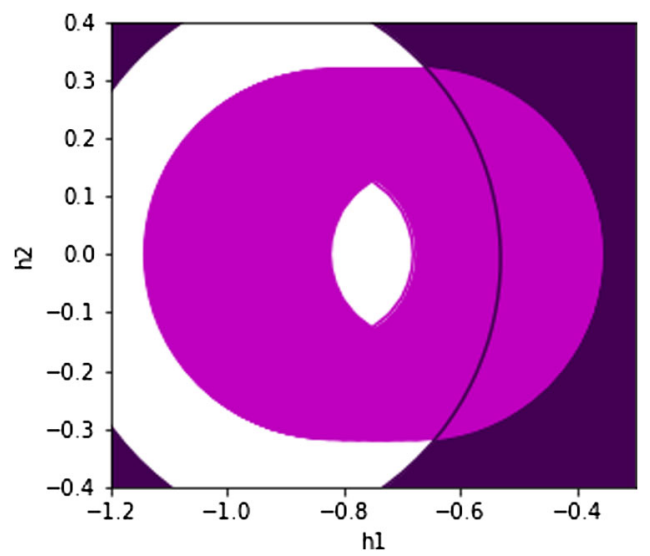

FIG. 1. Allowed range of $h_{1}^{I}$ and $h_{2}$. 
TABLE VI. Predicted widths of excited SHBs. We used the spin- and isospin-averaged value of the decay widths of $\Xi_{c}^{+}(2790), \Xi_{c}^{0}(2790), \Xi_{c}^{+}(2815)$, and $\Xi_{c}^{0}(2815)$ in addition to the decay width of $\Lambda_{c}(2595)$ as inputs.

\begin{tabular}{|c|c|c|c|c|c|}
\hline Initial & Mode & Our model (MeV) & [37] (MeV) & [25] (MeV) & Experimental $(\mathrm{MeV})$ \\
\hline \multirow{3}{*}{$\Lambda_{c}(2595)$} & $\Lambda_{c} \pi^{+} \pi^{-}$ & $0.562-1.09$ & & & \\
\hline & $\Lambda_{c} \pi^{0} \pi^{0}$ & $1.23-2.31$ & & & \\
\hline & Sum & 1.82-3.36 (input) & $\cdots$ & $\cdots$ & $2.59 \pm 0.30 \pm 0.47$ \\
\hline \multirow{3}{*}{$\Lambda_{c}(2625)$} & $\Lambda_{c} \pi^{+} \pi^{-}$ & $0.0618-0.507$ & & & \\
\hline & $\Lambda_{c} \pi^{0} \pi^{0}$ & $0.0431-0.226$ & & & \\
\hline & Sum & $0.106-0.733$ & $\cdots$ & $\cdots$ & $<0.97$ \\
\hline \multirow[t]{3}{*}{$\Lambda_{b}(5912)$} & $\Lambda_{b} \pi^{+} \pi^{-}$ & $(0.67-4.4) \times 10^{-3}$ & & & \\
\hline & $\Lambda_{b} \pi^{0} \pi^{0}$ & $(1.4-6.0) \times 10^{-3}$ & & & \\
\hline & Sum & $(2.1-10) \times 10^{-3}$ & $\cdots$ & $\cdots$ & $<0.66$ \\
\hline \multirow[t]{3}{*}{$\Lambda_{b}(5920)$} & $\Lambda_{b} \pi^{+} \pi^{-}$ & $(0.75-13) \times 10^{-3}$ & & & \\
\hline & $\Lambda_{b} \pi^{0} \pi^{0}$ & $(2.2-12) \times 10^{-3}$ & & & \\
\hline & Sum & $(3.0-25) \times 10^{-3}$ & $\cdots$ & $\cdots$ & $<0.63$ \\
\hline \multirow[t]{2}{*}{$\Xi_{c}^{+}(2790)$} & $\begin{array}{l}\Xi_{c}^{\prime+} \pi^{0} \\
\Xi_{c}^{\prime 0} \pi^{+}\end{array}$ & & & & \\
\hline & Sum & Input & $\cdots$ & 3.61 & $8.9 \pm 0.6 \pm 0.8$ \\
\hline \multirow[t]{3}{*}{$\Xi_{c}^{0}(2790)$} & $\Xi_{c}^{\prime 0} \pi^{0}$ & & & & \\
\hline & $\Xi_{c}^{\prime+} \pi^{-}$ & & & & \\
\hline & Sum & Input & $\cdots$ & 3.61 & $10.0 \pm 0.7 \pm 0.8$ \\
\hline \multirow[t]{3}{*}{$\Xi_{c}^{+}(2815)$} & $\Xi_{c}^{+}(2645) \pi^{0}$ & & & & \\
\hline & $\Xi_{c}^{0}(2645) \pi^{+}$ & & & & \\
\hline & Sum & Input & $\cdots$ & 1.80 & $2.43 \pm 0.20 \pm 0.17$ \\
\hline \multirow[t]{3}{*}{$\Xi_{c}^{0}(2815)$} & $\Xi_{c}^{0}(2645) \pi^{0}$ & & & & \\
\hline & $\Xi_{c}^{+}(2645) \pi^{-}$ & & & & \\
\hline & Sum & Input & $\cdots$ & 1.80 & $2.54 \pm 0.18 \pm 0.14$ \\
\hline \multirow[t]{3}{*}{$\Xi_{b 1}^{0}$} & $\Xi_{b}^{\prime 0} \pi^{0}$ & $0.0140-5.18$ & & & \\
\hline & $\Xi_{b}^{\prime-} \pi^{+}$ & $0.0275-10.1$ & & & \\
\hline & Sum & $0.0415-15.3$ & 4.2 & 2.84 & $\cdots$ \\
\hline \multirow[t]{3}{*}{$\Xi_{b 1}^{-}$} & $\Xi_{b}^{\prime-} \pi^{0}$ & $0.0140-5.18$ & & & \\
\hline & $\Xi_{b}^{\prime 0} \pi^{-}$ & $0.0275-10.1$ & & & \\
\hline & Sum & $0.0415-15.3$ & 4.2 & 2.84 & $\cdots$ \\
\hline \multirow[t]{3}{*}{$\Xi_{b 1}^{* 0}$} & $\Xi_{b}^{* 0} \pi^{0}$ & $0.109-4.31$ & & & \\
\hline & $\Xi_{b}^{*-} \pi^{+}$ & $<7.21$ & & & \\
\hline & Sum & $0.326-11.5$ & 2.9 & 2.88 & $\cdots$ \\
\hline \multirow[t]{3}{*}{$\Xi_{b 1}^{*-}$} & $\Xi_{b}^{*-} \pi^{0}$ & $0.109-4.31$ & & & \\
\hline & $\Xi_{b}^{* 0} \pi^{-}$ & $<7.21$ & & & \\
\hline & Sum & $0.326-11.5$ & 2.9 & 2.88 & $\cdots$ \\
\hline
\end{tabular}

$\Xi_{b 1}^{(*)}$ in Table VI are very small, and three-body decays such as $\Xi_{b 1} \rightarrow \Xi_{b} \pi \pi$ become dominant. Here, we study the contributions of possible intermediate states of threebody decays and show the results in Table VII, where we set the parameters as $g_{2}^{b}=0.980, \bar{\kappa}_{2}=0.807, g_{3}=0.688$, $h_{1}^{I}=-0.40$, and $h_{2}=0$, and set the masses of $\Xi_{b 1}^{*}$ to be their minimum of the predicted values shown in Table V, and $\Xi_{b}^{\prime *}$ to be the central mass values in Table I. We note that, unlike the decays of $\Lambda_{b}(5912)$ and $\Lambda_{b}(5920)$, the decays of $\Xi_{b 1}^{* 0}$ and $\Xi_{b 1}^{*-}$ are not dominated by the nonresonant contribution.

\section{RADIATIVE DECAYS}

In this section, we study radiative decays of the SHBs. The relevant Lagrangian is given by 
TABLE VII. Estimated values of the decay widths of bottom baryons.

\begin{tabular}{|c|c|c|c|}
\hline Initial state & Decay mode & Intermediate state & Width (keV) \\
\hline \multirow{9}{*}{$\Xi_{b 1}^{* 0}$} & \multirow[t]{3}{*}{$\Xi_{b}^{0} \pi^{0} \pi^{0}$} & nonresonant & 0.688 \\
\hline & & $\Xi_{b}^{\prime * 0}$ & 534 \\
\hline & & $\mathrm{NR} \& \Xi_{b}^{\prime * 0}$ & 2.97 \\
\hline & \multirow[t]{3}{*}{$\Xi_{b}^{0} \pi^{+} \pi^{-}$} & NR & 0.432 \\
\hline & & $\Xi_{b}^{\prime *-}$ & 8.67 \\
\hline & & $\mathrm{NR} \& \Xi_{b}^{*-}$ & 2.59 \\
\hline & \multirow[t]{3}{*}{$\Xi_{b}^{-} \pi^{+} \pi^{0}$} & $\Xi_{b}^{\prime * 0}$ & $2.44 \times 10^{3}$ \\
\hline & & $\Xi_{b}^{\prime *-}$ & 41.1 \\
\hline & & $\Xi_{b}^{\prime * 0} \& \Xi_{b}^{\prime *-}$ & 2.06 \\
\hline \multirow[t]{9}{*}{$\Xi_{b 1}^{*-}$} & \multirow[t]{3}{*}{$\Xi_{b}^{0} \pi^{0} \pi^{0}$} & NR & 0.510 \\
\hline & & $\Xi_{b}^{\prime *-}$ & 53.6 \\
\hline & & $\mathrm{NR} \& \Xi_{b}^{\prime *-}$ & 5.02 \\
\hline & \multirow[t]{3}{*}{$\Xi_{b}^{-} \pi^{+} \pi^{-}$} & NR & 0.275 \\
\hline & & $\Xi_{b}^{\prime * 0}$ & 194 \\
\hline & & $\mathrm{NR} \& \Xi_{b}^{\prime * 0}$ & 4.55 \\
\hline & \multirow[t]{3}{*}{$\Xi_{b}^{0} \pi^{-} \pi^{0}$} & $\Xi_{b}^{\prime *-}$ & 386 \\
\hline & & $\Xi_{b}^{\prime * 0}$ & $1.40 \times 10^{3}$ \\
\hline & & $\Xi_{b}^{\prime *-} \& \Xi_{b}^{\prime * 0}$ & 5.39 \\
\hline
\end{tabular}

$$
\begin{aligned}
\mathcal{L}_{\text {rad }}= & \frac{r_{1}}{F} \operatorname{tr}\left(\bar{S}_{Q}^{\mu} Q_{\text {light }} S_{Q}^{\nu}+\bar{S}_{Q}^{\mu T} Q_{\text {light }} S_{Q}^{\nu T}\right) F_{\mu \nu} \\
& +\frac{r_{2}}{F} \operatorname{tr}\left(\bar{S}_{Q}^{\mu} Q_{\text {light }} S_{Q}^{\nu}-\bar{S}_{Q}^{\mu T} Q_{\text {light }} S_{Q}^{\nu T}\right) \tilde{F}_{\mu \nu} \\
& +\frac{r_{3}}{F^{2}} \operatorname{tr}\left(\bar{S}_{Q L L} M S_{Q}^{\mu} Q_{\text {light }} v^{\nu}+\bar{S}_{Q R R} M^{\dagger} S_{Q}^{\mu} Q_{\text {light }} v^{\nu}\right) F_{\mu \nu} \\
& + \text { H.c. } \\
& +\frac{r_{4}}{F^{2}} \operatorname{tr}\left(\bar{S}_{Q L L} M S_{Q}^{\mu} Q_{\text {light }} v^{\nu}+\bar{S}_{Q R R} M^{\dagger} S_{Q}^{\mu} Q_{\text {light }} v^{\nu}\right) \tilde{F}_{\mu \nu} \\
& + \text { H.c. },
\end{aligned}
$$

where $F_{\mu \nu}$ is the field strength of the photon and $\tilde{F}_{\mu \nu}$ is its dual tensor: $\tilde{F}_{\mu \nu}=(1 / 2) \epsilon_{\mu \nu \rho \sigma} F^{\rho \sigma}, \quad r_{i}(i=1, \ldots, 4)$ are dimensionless constants, and $F$ is a constant with dimension one. In this analysis, we take $F=350 \mathrm{MeV}$ following Ref. [17]. We note that the values of the constants $r_{i}$ are of order one based on quark models [17].

Let us first study the electromagnetic intramultiplet transitions governed by the $r_{1}$-term in Eq. (32). Let $B^{*}$ denote the decaying baryon with spin-3/2 $\left(B^{*}=\Lambda_{Q 1}^{*}\right.$, $\left.\Xi_{Q 1}^{*}, \Sigma_{Q}^{*}, \Xi_{Q}^{* *}, \Omega_{Q}^{*}\right)$, and $B$, the daughter baryon with spin-1/2 $\left(\Lambda_{Q 1}, \Xi_{Q 1}, \Sigma_{Q}, \Xi_{Q}^{\prime}, \Omega_{Q}\right)$. Then the radiative decay width is given by

$$
\Gamma_{B^{*} \rightarrow B \gamma}=C_{B^{*} B \gamma}^{2} \frac{16 \alpha r_{1}^{2}}{9 F^{2}} \frac{m_{B}}{m_{B^{*}}} E_{\gamma}^{3}
$$

where $\alpha$ is the electromagnetic fine structure constant, $E_{\gamma}$ is the photon energy, and $C_{B^{*} B \gamma}$ is the Clebsh-Gordon constants given by

$$
\begin{gathered}
C_{\Sigma_{c}^{*++} \Sigma_{c}^{++} \gamma}=C_{\Sigma_{b}^{*+} \Sigma_{b}^{+} \gamma}=\frac{2}{3}, \\
C_{\Sigma_{c}^{*+} \Sigma_{c}^{+} \gamma}=C_{\Sigma_{b}^{* 0} \Sigma_{b}^{0} \gamma}=\frac{1}{6}, \\
C_{\Sigma_{c}^{* 0} \Sigma_{c}^{0} \gamma}=C_{\Sigma_{b}^{*-} \Sigma_{b}^{-} \gamma}=-\frac{1}{3}, \\
C_{\Xi_{c}^{*+} \Xi_{c}^{+} \gamma}=C_{\Xi_{b}^{* 0} \Xi_{b}^{0} \gamma}=\frac{1}{6}, \\
C_{\Xi_{c}^{* 0} \Xi_{c}^{0} \gamma}=C_{\Xi_{b}^{*-} \Xi_{b}^{-} \gamma}=-\frac{1}{3}, \\
C_{\Omega_{c}^{* 0} \Omega_{c}^{0} \gamma}=C_{\Omega_{b}^{*-} \Omega_{b}^{-} \gamma}=-\frac{1}{3}, \\
C_{\Lambda_{c 1}^{*+} \Lambda_{c 1}^{+} \gamma}=C_{\Lambda_{b}^{* 0} \Lambda_{b}^{0} \gamma}=-\frac{1}{6}, \\
C_{\Xi_{c 1}^{*+} \Xi_{c 1}^{+} \gamma}=C_{\Xi_{b 1}^{* 0} \Xi_{b 1}^{0} \gamma}=-\frac{1}{6}, \\
C_{\Xi_{c 1}^{* 0} \Xi_{c 1}^{0} \gamma}=C_{\Xi_{b 1}^{*-} \Xi_{b 1}^{-} \gamma}=\frac{1}{3} .
\end{gathered}
$$

Here we would like to stress that the radiative decay widths of positive parity SHBs $\left(\Sigma_{Q}^{*}, \Xi_{Q}^{*}, \Omega_{Q}^{*}\right)$ and those of negative parity SHBs $\left(\Lambda_{Q 1}^{*}, \Xi_{Q 1}^{*}\right)$ are determined by just one coupling constant $r_{1}$, reflecting the chiral partner structure. We think that checking the relation among these radiative decays will be one of the crucial tests of the chiral partner structure. In Tables VIII and IX, we show our predictions compared with those in Refs. [18,32]. In radiative decays, the chiral loop considered in Ref. [18] might have a contribution. However, our predictions are consistent with those in Ref. [18] for $r_{1} \sim 1$, which implies the contribution from the chiral loop is small in this radiative decay. On the other hand, our results are consistent with the lattice results if $r_{1} \sim 0.2$.

We note that $\Omega_{Q}^{*}$ does not have any strong decays and the main mode must be $\Omega_{Q}^{*} \rightarrow \Omega_{Q} \gamma$. We expect the coupling

TABLE VIII. Predicted widths of radiative decays between heavy-quark multiplets of charm baryons. We also show the predictions in Refs. [18,32] for comparison.

\begin{tabular}{lccc}
\hline \hline Decay mode & Predicted width $(\mathrm{keV})$ & {$[18](\mathrm{keV})$} & {$[32](\mathrm{keV})$} \\
\hline$\Sigma_{c}^{*++} \rightarrow \Sigma_{c}^{++} \gamma$ & $11.8 r_{1}^{2}$ & 11.6 & $\ldots$ \\
$\Sigma_{c}^{*+} \rightarrow \Sigma_{c}^{+} \gamma$ & $0.743 r_{1}^{2}$ & 0.85 & $\ldots$ \\
$\Sigma_{c}^{* 0} \rightarrow \Sigma_{c}^{0} \gamma$ & $2.99 r_{1}^{2}$ & 2.92 & $\ldots$ \\
$\Xi_{c}^{\prime+} \rightarrow \Xi_{c}^{\prime+} \gamma$ & $0.872 r_{1}^{2}$ & 1.10 & $\ldots$ \\
$\Xi_{c}^{* 0} \rightarrow \Xi_{c}^{\prime 0} \gamma$ & $3.40 r_{1}^{2}$ & 3.83 & $\ldots$ \\
$\Omega_{c}^{* 0} \rightarrow \Omega_{c}^{0} \gamma$ & $3.90 r_{1}^{2}$ & 4.82 & $0.096(14)$ \\
$\Lambda_{c 1}^{*+} \rightarrow \Lambda_{c 1}^{+} \gamma$ & $0.131 r_{1}^{2}$ & $\ldots$ & $\ldots$ \\
$\Xi_{c 1}^{*+} \rightarrow \Xi_{c 1}^{+} \gamma$ & $0.0432 r_{1}^{2}$ & $\ldots$ & $\ldots$ \\
$\Xi_{c 1}^{* 0} \rightarrow \Xi_{c 1}^{0} \gamma$ & $0.237 r_{1}^{2}$ & $\ldots$ & $\ldots$ \\
\hline \hline
\end{tabular}


TABLE IX. Predicted widths of radiative decays between heavy-quark multiplets of bottom baryons. We also show the predictions in Ref. [18] for comparison.

\begin{tabular}{lcc}
\hline \hline Decay mode & Predicted width $(\mathrm{keV})$ & {$[18](\mathrm{keV})$} \\
\hline$\Sigma_{b}^{*+} \rightarrow \Sigma_{b}^{+} \gamma$ & $0.420 r_{1}^{2}$ & 0.60 \\
$\Sigma_{b}^{* 0} \rightarrow \Sigma_{b}^{0} \gamma$ & $0.0240 r_{1}^{2}$ & 0.05 \\
$\Sigma_{b}^{*-} \rightarrow \Sigma_{b}^{-} \gamma$ & $0.0879 r_{1}^{2}$ & 0.08 \\
$\Xi_{b}^{\prime * 0} \rightarrow \Xi_{b}^{\prime 0} \gamma$ & $0.00944 r_{1}^{2}$ & $\ldots$ \\
$\Xi_{b}^{\prime *-} \rightarrow \Xi_{b}^{\prime-} \gamma$ & $0.0977 r_{1}^{2}$ & $\ldots$ \\
$\Omega_{b}^{*-} \rightarrow \Omega_{b}^{-} \gamma$ & $0.0920-4.07 r_{1}^{2}$ & $\ldots$ \\
$\Lambda_{b 1}^{* 0} \rightarrow \Lambda_{b 1}^{0} \gamma$ & $0.00135 r_{1}^{2}$ & $\ldots$ \\
$\Xi_{b 1}^{* 0} \rightarrow \Xi_{b 1}^{0} \gamma$ & $<0.453 r_{1}^{2}$ & $\ldots$ \\
$\Xi_{b 1}^{*-} \rightarrow \Xi_{b 1}^{-} \gamma$ & $<1.81 r_{1}^{2}$ & $\ldots$ \\
\hline \hline
\end{tabular}

constant $r_{1}$ will be determined by the decay of $\Omega_{Q}^{*}$ in future experiments, and other radiative decay widths related to $r_{1^{-}}$ type interaction will be estimated.

We next study the radiative decays between the SHBs with negative parity in the flavor $\mathbf{3}$ representations and the SHBs with positive parity in the flavor 6 representations, which concern the $r_{2}$-term. The decay widths are expressed as

$$
\begin{gathered}
\Gamma_{\Lambda_{Q 1} \rightarrow \Sigma_{Q \gamma}}=\frac{16 \alpha r_{2}^{2}}{9 F^{2}} \frac{m_{\Sigma_{Q}}}{m_{\Lambda_{Q 1}}} E_{\gamma}^{3}, \\
\Gamma_{\Lambda_{Q 1} \rightarrow \Sigma_{Q}^{*} \gamma}=\frac{8 \alpha r_{2}^{2}}{9 F^{2}} \frac{m_{\Sigma_{Q}^{*}}}{m_{\Lambda_{Q 1}}} E_{\gamma}^{3}, \\
\Gamma_{\Lambda_{Q 1}^{*} \rightarrow \Sigma_{Q \gamma}}=\frac{4 \alpha r_{2}^{2}}{9 F^{2}} \frac{m_{\Sigma_{Q}}}{m_{\Lambda_{Q 1}^{*}}} E_{\gamma}^{3}, \\
\Gamma_{\Lambda_{Q 1}^{*} \rightarrow \Sigma_{Q}^{*} \gamma}=\frac{20 \alpha r_{2}^{2}}{9 F^{2}} \frac{m_{\Sigma_{Q}^{*}}}{m_{\Lambda_{Q 1}^{*}}} E_{\gamma}^{3} \\
\Gamma_{\Xi_{Q 1}^{+} \rightarrow \Xi_{Q}^{\prime+\gamma}}=\frac{16 \alpha r_{2}^{2}}{9 F^{2}} \frac{m_{\Xi_{Q}^{\prime+}}}{m_{\Xi_{Q 1}^{+}}} E_{\gamma}^{3}, \\
\Gamma_{\Xi_{Q 1}^{+} \rightarrow \Xi_{Q}^{\prime *+} \gamma}=\frac{8 \alpha r_{2}^{2}}{9 F^{2}} \frac{m_{\Xi_{Q}^{\prime \prime}}}{m_{\Xi_{Q 1}}} E_{\gamma}^{3}, \\
\Gamma_{\Xi_{Q 1}^{*+} \rightarrow \Xi_{Q}^{\prime+} \gamma}=\frac{4 \alpha r_{2}^{2}}{9 F^{2}} \frac{m_{\Xi_{Q}^{\prime+}}}{m_{\Xi_{Q 1}^{*+}}^{*+}} E_{\gamma}^{3}, \\
\Gamma_{\Xi_{Q 1}^{*+} \rightarrow \Xi_{Q}^{\prime *+} \gamma}=\frac{20 \alpha r_{2}^{2}}{9 F^{2}} \frac{m_{\Xi_{Q}^{\prime *}}}{m_{\Xi_{Q 1}^{*+}}} E_{\gamma}^{3} .
\end{gathered}
$$

In Tables $\mathrm{X}$ and $\mathrm{XI}$, we show our predictions compared with those in Refs. [17,20]. Our results are consistent with those in Ref. [17] when $r_{2} \sim c_{R S} / \sqrt{2}$, and with those in Ref. [20] when $r_{2} \sim 1 / 2$.
TABLE X. Predicted widths of radiative decays between negative parity charm baryons in the flavor $\mathbf{3}$ representations and positive parity charm baryons in the flavor $\mathbf{6}$ representations. We also show the predictions in Ref. [17,20] for comparison.

\begin{tabular}{lccc}
\hline \hline Decay mode & Predicted width (keV) & {$[17](\mathrm{keV})$} & {$[20](\mathrm{keV})$} \\
\hline$\Lambda_{c 1}^{+} \rightarrow \Sigma_{c}^{+} \gamma$ & $250 r_{2}^{2}$ & $127 c_{R S}^{2}$ & $77 \pm 1$ \\
$\Lambda_{c 1}^{+} \rightarrow \Sigma_{c}^{*+} \gamma$ & $20.6 r_{2}^{2}$ & $6 c_{R S}^{2}$ & $6 \pm 0.1$ \\
$\Lambda_{c 1}^{*+} \rightarrow \Sigma_{c}^{+} \gamma$ & $120 r_{2}^{2}$ & $58 c_{R S}^{2}$ & $35 \pm 0.5$ \\
$\Lambda_{c 1}^{*+} \rightarrow \Sigma_{c}^{*+} \gamma$ & $161 r_{2}^{2}$ & $54 c_{R S}^{2}$ & $46 \pm 0.6$ \\
$\Xi_{c 1}^{+} \rightarrow \Xi_{c}^{\prime+} \gamma$ & $859 r_{2}^{2}$ & $\ldots$ & $\ldots$ \\
$\Xi_{c 1}^{+} \rightarrow \Xi_{c}^{*+} \gamma$ & $146 r_{2}^{2}$ & $\ldots$ & $\ldots$ \\
$\Xi_{c 1}^{*+} \rightarrow \Xi_{c}^{\prime+} \gamma$ & $291 r_{2}^{2}$ & $\ldots$ & $\ldots$ \\
$\Xi_{c 1}^{*+} \rightarrow \Xi_{c}^{\prime *+} \gamma$ & $568 r_{2}^{2}$ & $\ldots$ & $\ldots$ \\
\hline \hline
\end{tabular}

The $r_{3}$-term generates the radiative decays between the negative parity SHBs in the flavor 3 representations and the positive parity SHBs in the flavor $\mathbf{3}$ representations, the widths of which are expressed as

$$
\begin{aligned}
\Gamma_{\Lambda_{Q 1}^{(*)} \rightarrow \Lambda_{Q \gamma} \gamma} & =\frac{8 \alpha r_{3}^{2} f_{\pi}^{2}}{27 F^{4}} \frac{m_{\Lambda_{Q}}}{m_{\Lambda_{Q 1}^{(*)}}} E_{\gamma}^{3}, \\
\Gamma_{\Xi_{Q 1}^{(*)+} \rightarrow \Xi_{Q}^{+} \gamma} & =\frac{8 \alpha r_{3}^{2}\left(f_{\pi}-2 \sigma_{s}\right)^{2}}{27 F^{4}} \frac{m_{\Xi_{Q}^{+}}}{m_{\Xi_{Q 1}^{(*)+}}} E_{\gamma}^{3}, \\
\Gamma_{\Xi_{Q 1}^{(*)} \rightarrow \Xi_{Q}^{0} \gamma} & =\frac{8 \alpha r_{3}^{2}\left(f_{\pi}+\sigma_{s}\right)^{2}}{27 F^{4}} \frac{m_{\Xi_{Q}}^{0}}{m_{\Xi_{Q 1}^{(*) 0}}} E_{\gamma}^{3} .
\end{aligned}
$$

In Tables XII and XIII, we show our predictions together with the ones in Refs. [17,20]. We think that the differences between our predictions and those in Ref. [20] are from the value of $\sigma_{s}$ : we use $\sigma_{s}=2 f_{K}-f_{\pi}$ while $\sigma_{s}=f_{\pi}$ is used in Ref. [20].

The widths of radiative decays between the positive parity SHBs in the flavor 6 representations and the positive

TABLE XI. Predicted widths of radiative decays between negative parity bottom baryons in the flavor 3 representations and positive parity bottom baryons in the flavor $\mathbf{6}$ representations.

\begin{tabular}{lc}
\hline \hline Decay mode & Predicted width $(\mathrm{keV})$ \\
\hline$\Lambda_{b 1}^{0} \rightarrow \Sigma_{b}^{0} \gamma$ & $97.9 r_{2}^{2}$ \\
$\Lambda_{b 1}^{0} \rightarrow \Sigma_{b}^{* 0} \gamma$ & $24.8 r_{2}^{2}$ \\
$\Lambda_{b 1}^{* 0} \rightarrow \Sigma_{b}^{0} \gamma$ & $30.6 r_{2}^{2}$ \\
$\Lambda_{b 1}^{* 0} \rightarrow \Sigma_{b}^{* 0} \gamma$ & $82.0 r_{2}^{2}$ \\
$\Xi_{b 1}^{0} \rightarrow \Xi_{b}^{\prime 0} \gamma$ & $370-887 r_{2}^{2}$ \\
$\Xi_{b 1}^{0} \rightarrow \Xi_{b}^{\prime * 0} \gamma$ & $138-358 r_{2}^{2}$ \\
$\Xi_{b 1}^{* 0} \rightarrow \Xi_{b}^{\prime 0} \gamma$ & $92.4-222 r_{2}^{2}$ \\
$\Xi_{b 1}^{* 0} \rightarrow \Xi_{b}^{\prime * 0} \gamma$ & $344-895 r_{2}^{2}$ \\
\hline \hline
\end{tabular}


TABLE XII. Predicted widths of radiative decays between negative parity charm baryons in the flavor $\mathbf{3}$ representations and positive parity charm baryons in the flavor $\mathbf{3}$ representations. We also show the predictions in Ref. [17,20].

\begin{tabular}{lccc}
\hline \hline Decay mode & Predicted width $(\mathrm{keV})$ & {$[17](\mathrm{keV})$} & {$[20](\mathrm{keV})$} \\
\hline$\Lambda_{c 1} \rightarrow \Lambda_{c} \gamma$ & $25.9 r_{3}^{2}$ & $191 c_{R T}^{s}$ & $115 \pm 1$ \\
$\Lambda_{c 1}^{*} \rightarrow \Lambda_{c} \gamma$ & $34.9 r_{3}^{2}$ & $253 c_{R T}^{2}$ & $151 \pm 2$ \\
$\Xi_{c 1}^{+} \rightarrow \Xi_{c}^{+} \gamma$ & $98.9 r_{3}^{2}$ & $\ldots$ & $\ldots$ \\
$\Xi_{c 1}^{*+} \rightarrow \Xi_{c}^{+} \gamma$ & $121 r_{3}^{2}$ & $\ldots$ & $190 \pm 5$ \\
$\Xi_{c 1}^{0} \rightarrow \Xi_{c}^{0} \gamma$ & $174 r_{3}^{2}$ & $\ldots$ & $\ldots$ \\
$\Xi_{c 1}^{* 0} \rightarrow \Xi_{c}^{0} \gamma$ & $217 r_{3}^{2}$ & $\ldots$ & $497 \pm 14$ \\
\hline \hline
\end{tabular}

TABLE XIII. Predicted widths of radiative decays between negative parity bottom baryons in the flavor 3 representations and positive parity bottom baryons in the flavor $\mathbf{3}$ representations.

\begin{tabular}{cc}
\hline \hline Decay mode & Predicted width $(\mathrm{keV})$ \\
\hline$\Lambda_{b 1} \rightarrow \Lambda_{b} \gamma$ & $27.2 r_{3}^{2}$ \\
$\Lambda_{b 1}^{*} \rightarrow \Lambda_{b} \gamma$ & $29.3 r_{3}^{2}$ \\
$\Xi_{b 1}^{0} \rightarrow \Xi_{b}^{0} \gamma$ & $92.0-148 r_{3}^{2}$ \\
$\Xi_{b 1}^{* 0} \rightarrow \Xi_{b}^{0} \gamma$ & $92.0-148 r_{3}^{2}$ \\
$\Xi_{b 1}^{-} \rightarrow \Xi_{b}^{-} \gamma$ & $161-260 r_{3}^{2}$ \\
$\Xi_{b 1}^{*-} \rightarrow \Xi_{b}^{-} \gamma$ & $161-260 r_{3}^{2}$ \\
\hline \hline
\end{tabular}

parity SHBs in the flavor 3 representations via the $r_{4}$-term are given by

$$
\begin{aligned}
\Gamma_{\Sigma_{Q}^{(*)} \rightarrow \Lambda_{Q} \gamma} & =\frac{8 \alpha r_{4}^{2} f_{\pi}^{2}}{3 F^{4}} \frac{m_{\Lambda_{Q}}}{m_{\Sigma_{Q}^{(*)}}} E_{\gamma}^{3}, \\
\Gamma_{\Xi_{Q}^{(*)+} \rightarrow \Xi_{Q}^{+} \gamma} & =\frac{8 \alpha r_{4}^{2}\left(f_{\pi}+2 \sigma_{s}\right)^{2}}{27 F^{4}} \frac{m_{\Xi_{Q}^{+}}}{m_{\Xi_{Q}^{\prime(*)+}}} E_{\gamma}^{3}, \\
\Gamma_{\Xi_{Q}^{\prime(*)} \rightarrow \Xi_{Q}^{0} \gamma} & =\frac{8 \alpha r_{4}^{2}\left(f_{\pi}-\sigma_{s}\right)^{2}}{27 F^{4}} \frac{m_{\Xi_{Q}^{0}}}{m_{\Xi_{Q}^{\prime(*) 0}}} E_{\gamma}^{3},
\end{aligned}
$$

TABLE XIV. Predicted widths of radiative decays between positive parity charm baryons in the flavor $\mathbf{6}$ representations and positive parity charm baryons in the flavor 3 representations. We also show the predictions in Refs. [18,31].

\begin{tabular}{lccc}
\hline \hline Decay mode & Predicted width $(\mathrm{keV})$ & [18] $(\mathrm{keV})$ & [31] $(\mathrm{keV})$ \\
\hline$\Sigma_{c}^{+} \rightarrow \Lambda_{c}^{+} \gamma$ & $42.9 r_{4}^{2}$ & 164 & $\ldots$ \\
$\Sigma_{c}^{*+} \rightarrow \Lambda_{c}^{+} \gamma$ & $108 r_{4}^{2}$ & 893 & $\ldots$ \\
$\Xi_{c}^{\prime+} \rightarrow \Xi_{c}^{+} \gamma$ & $20.8 r_{4}^{2}$ & 54.3 & $5.468(1.500)$ \\
$\Xi_{c}^{\prime *+} \rightarrow \Xi_{c}^{+} \gamma$ & $83.3 r_{4}^{2}$ & 502 & $\ldots$ \\
$\Xi_{c}^{\prime 0} \rightarrow \Xi_{c}^{0} \gamma$ & $0.216 r_{4}^{2}$ & 0.02 & $0.002(4)$ \\
$\Xi_{c}^{\prime * 0} \rightarrow \Xi_{c}^{0} \gamma$ & $0.870 r_{4}^{2}$ & 0.36 & $\ldots$ \\
\hline \hline
\end{tabular}

TABLE XV. Predicted widths of radiative decays between positive parity bottom baryons in the flavor $\mathbf{6}$ representations and positive parity bottom baryons in the flavor 3 representations. We also show the predictions in Ref. [18].

\begin{tabular}{lcc}
\hline \hline Decay mode & Predicted width $(\mathrm{keV})$ & {$[18](\mathrm{keV})$} \\
\hline$\Sigma_{b}^{0} \rightarrow \Lambda_{b}^{0} \gamma$ & $74.1 r_{4}^{2}$ & 288 \\
$\Sigma_{b}^{* 0} \rightarrow \Lambda_{b}^{0} \gamma$ & $98.9 r_{4}^{2}$ & 435 \\
$\Xi_{b}^{\prime 0} \rightarrow \Xi_{b}^{0} \gamma$ & $48.7 r_{4}^{2}$ & $\ldots$ \\
$\Xi_{b}^{\prime * 0} \rightarrow \Xi_{b}^{0} \gamma$ & $65.0 r_{4}^{2}$ & 136 \\
$\Xi_{b}^{\prime-} \rightarrow \Xi_{b}^{-} \gamma$ & $0.499 r_{4}^{2}$ & $\ldots$ \\
$\Xi_{b}^{*-} \rightarrow \Xi_{b}^{-} \gamma$ & $0.742 r_{4}^{2}$ & 1.87 \\
\hline \hline
\end{tabular}

and the predicted values are shown in Tables XIV and XV with the ones in Refs. [18,31]. Our results are consistent with the lattice results in Ref. [31] if $r_{4} \sim 0.1$. On the other

\begin{tabular}{|c|c|c|c|c|}
\hline SHB & $J^{P}$ & $\begin{array}{l}\text { Decay } \\
\text { modes }\end{array}$ & Our model (MeV) & $\begin{array}{c}\text { Experimental } \\
(\mathrm{MeV})\end{array}$ \\
\hline$\Lambda_{b 1}$ & $1 / 2^{-}$ & $\begin{array}{c}\Lambda_{b} \pi^{+} \pi^{-} \\
\Lambda_{b} \pi^{0} \pi^{0} \\
\Sigma_{b}^{0} \gamma \\
\Sigma_{b}^{* 0} \gamma \\
\Lambda_{b} \gamma\end{array}$ & $\begin{array}{c}(0.67-4.4) \times 10^{-3} \\
(1.4-6.0) \times 10^{-3} \\
0.098 r_{2}^{2} \\
0.025 r_{2}^{2} \\
0.027 r_{3}^{2}\end{array}$ & $<0.66$ \\
\hline$\Lambda_{b 1}^{*}$ & $3 / 2^{-}$ & $\begin{array}{c}\Lambda_{b} \pi^{+} \pi^{-} \\
\Lambda_{b} \pi^{0} \pi^{0} \\
\Lambda_{b 1} \gamma \\
\Sigma_{b}^{0} \gamma \\
\Sigma_{b}^{* 0} \gamma \\
\Lambda_{b} \gamma\end{array}$ & $\begin{array}{c}(0.75-13) \times 10^{-3} \\
(2.2-12) \times 10^{-3} \\
0.0013 r_{1}^{2} \times 10^{-3} \\
0.031 r_{2}^{2} \\
0.081 r_{2}^{2} \\
0.029 r_{3}^{2}\end{array}$ & $<0.63$ \\
\hline$\Xi_{b 1}^{0}$ & $1 / 2^{-}$ & $\begin{array}{l}\Xi_{b}^{\prime} \pi \\
\Xi_{b}^{\prime 0} \gamma \\
\Xi_{b}^{\prime * 0} \gamma \\
\Xi_{b}^{0} \gamma\end{array}$ & $\begin{array}{c}0.0415-15.3 \\
0.370-0.887 r_{2}^{2} \\
0.138-0.358 r_{2}^{2} \\
0.0288-0.0464 r_{3}^{2}\end{array}$ & $\cdots$ \\
\hline$\Xi_{b 1}^{-}$ & $1 / 2^{-}$ & $\begin{array}{l}\Xi_{b}^{\prime} \pi \\
\Xi_{b}^{\prime-} \gamma \\
\Xi_{b}^{\prime *-} \gamma \\
\Xi_{b}^{-} \gamma\end{array}$ & $\begin{array}{c}0.0415-15.3 \\
\ldots \\
\cdots \\
0.112-0.182 r_{3}^{2}\end{array}$ & $\cdots$ \\
\hline$\Xi_{b 1}^{* 0}$ & $3 / 2^{-}$ & $\begin{array}{l}\Xi_{b}^{\prime} \pi \\
\Xi_{b 1}^{0} \gamma \\
\Xi_{b}^{\prime 0} \gamma \\
\Xi_{b}^{\prime * 0} \gamma \\
\Xi_{b}^{0} \gamma\end{array}$ & $\begin{array}{c}0.326-11.5 \\
<4.53 r_{1}^{2} \times 10^{-4} \\
0.0924-0.222 r_{2}^{2} \\
0.344-0.895 r_{2}^{2} \\
0.0288-0.0464 r_{3}^{2}\end{array}$ & $\cdots$ \\
\hline$\Xi_{b 1}^{*-}$ & $3 / 2^{-}$ & $\begin{array}{l}\Xi_{b}^{\prime} \pi \\
\Xi_{b 1}^{-} \gamma \\
\Xi_{b}^{\prime-} \gamma \\
\Xi_{b}^{\prime *-} \gamma \\
\Xi_{b}^{-} \gamma\end{array}$ & $\begin{array}{c}0.326-11.5 \\
<1.81 r_{1}^{2} \times 10^{-3} \\
\cdots \\
\cdots \\
0.112-0.182 r_{3}^{2}\end{array}$ & $\cdots$ \\
\hline
\end{tabular}

TABLE XVI. Pionic and radiative decays of bottom SHBs with negative parity. 
hand, a comparison with the results in Ref. [18] indicates that the chiral loop may be important.

\section{A SUMMARY AND DISCUSSIONS}

We constructed an effective hadronic model regarding negative parity $\mathbf{3}$ representations as chiral partners to positive parity 6 representations, based on the chiral symmetry and heavy-quark spin-flavor symmetry. We determine the model parameters from the experimental data for relevant masses and decay widths of $\Sigma_{c}\left(2455,1 / 2^{+}\right)$, $\Sigma_{c}\left(2520,3 / 2^{+}\right), \quad \Lambda_{c}\left(2595,1 / 2^{-}\right), \quad \Xi_{c}\left(2790,1 / 2^{-}\right)$, and $\Xi_{c}\left(2815,1 / 2^{-}\right)$. Then, we studied the decay widths of $\Lambda_{c}(2625), \Lambda_{b}(5912), \Lambda_{b}(5920)$, and negative parity $\Xi_{b}^{(*)}$, which have not been yet discovered in any experiments. We think that $\Xi_{b}^{(*)}$ here is unlikely to be $\Xi_{b}(6227)$ reported in Ref. [38], which may be explained as, e.g., a molecule state in Ref. [39]. Using the model parameters, we predict the values for masses and decay widths of negative parity excited $\Xi_{b}$.

As shown in our previous work, Ref. [12], the chiral partner structure is reflected in the direct decay processes in three-body decays of negative parity 3 representations. Our results for the three-body decays of $\Xi_{b 1}^{*} \rightarrow \Xi_{b} \pi \pi$ are dominated by the resonant decay modes unlikely to the decays of $\Lambda_{c}(2625), \Lambda_{b}(5912)$, and $\Lambda_{b}(5920)$ shown in Ref. [12]. However, the Dalitz analysis, which was performed in Ref. [13] for the decays of $\Lambda_{c} \mathrm{~s}$ and $\Lambda_{b} \mathrm{~s}$, may give information of the direct decays of $\Xi$ s. Therefore, we would like to stress that future investigations of detailed threebody decay processes of negative parity SHBs will provide some clues to understand the chiral partner structure.

We also studied the radiative decays of the SHBs included in the present model using the effective interaction
Lagrangians in Eq. (32). We showed that there is a relation among the radiative decay widths of positive parity SHBs $\left(\Sigma_{Q}^{*}, \Xi_{Q}^{*}, \Omega_{Q}^{*}\right)$ and those of negative parity $\operatorname{SHBs}\left(\Lambda_{Q 1}^{*}\right.$, $\Xi_{Q 1}^{*}$ ), reflecting the chiral partner structure. Since the masses of negative parity SHBs in the bottom sector are close to the threshold of hadronic decays, the radiative decay widths can be comparable with the strong decay widths depending on the precise values of the masses. We summarize the decays of bottom SHBs with negative parity in Table XVI.

We expect that experimental study of these radiative decays will provide a clue to understand the chiral partner structure. In addition, we predict the $\Omega_{Q}^{(*)} \rightarrow \Omega_{Q} \gamma$ decay which is the sole decay mode of $\Omega_{Q}^{(*)}$. Experimental observation of this in the future will be a check of the present framework based on the effective model respecting the chiral symmetry and the heavy-quark spin-flavor symmetry. In addition, we expect that the future lattice simulations for the radiative decay of negative parity SHBs also provide some clues to the chiral partner structure.

\section{ACKNOWLEDGMENTS}

We would like to thank Professor Veljko Dmitrašinović, Professor Hua-Xing Chen, and Professor Atsushi Hosaka for their useful comments. The work of M. H. is supported in part by JSPS KAKENHI Grant No. 16K05345.

Note added.-While we are writing this manuscript, we are informed of Ref. [40], in which the chiral partner structure of SHBs is studied based on the mirror assignment of parity doublet structure including three chiral representations, i.e., $(\mathbf{3}, \mathbf{3}),(\overline{\mathbf{3}}, \mathbf{1})+(\mathbf{1}, \overline{\mathbf{3}})$, and $(\mathbf{6}, \mathbf{1})+(\mathbf{1}, \mathbf{6})$.
[1] M. A. Nowak, M. Rho, and I. Zahed, Chiral effective action with heavy quark symmetry, Phys. Rev. D 48, 4370 (1993).

[2] M. A. Nowak and I. Zahed, Excited heavy mesons, Phys. Rev. D 48, 356 (1993).

[3] W. A. Bardeen and C. T. Hill, Chiral dynamics and heavy quark symmetry in a solvable toy field theoretic model, Phys. Rev. D 49, 409 (1994).

[4] W. A. Bardeen, E. J. Eichten, and C. T. Hill, Chiral multiplets of heavy-light mesons, Phys. Rev. D 68, 054024 (2003).

[5] M. A. Nowak, M. Rho, and I. Zahed, Chiral doubling of heavy light hadrons: BABAR 2317-MeV/ $c^{2}$ and CLEO $2463-\mathrm{MeV} / c^{2}$ discoveries, Acta Phys. Pol. B 35, 2377 (2004).

[6] Y. L. Ma and M. Harada, Doubly heavy baryons with chiral partner structure, Phys. Lett. B 748, 463 (2015).
[7] Y. L. Ma and M. Harada, Degeneracy of doubly heavy baryons from heavy quark symmetry, Phys. Lett. B 754, 125 (2016).

[8] Y. L. Ma and M. Harada, Chiral partner structure of doubly heavy baryons with heavy quark spin-flavor symmetry, J. Phys. G 45, 075006 (2018).

[9] M. A. Nowak, M. Praszalowicz, M. Sadzikowski, and J. Wasiluk, Chiral doublers of heavy light baryons, Phys. Rev. D 70, 031503 (2004).

[10] M. Harada and Y. L. Ma, Chiral partner structure of heavy baryons from the bound state approach with hidden local symmetry, Phys. Rev. D 87, 056007 (2013).

[11] Y. R. Liu and M. Oka, $\Lambda_{c} N$ bound states revisited, Phys. Rev. D 85, 014015 (2012).

[12] Y. Kawakami and M. Harada, Analysis of $\Lambda_{c}(2595)$, $\Lambda_{c}(2625), \Lambda_{b}(5912), \Lambda_{b}(5920)$ based on a chiral partner structure, Phys. Rev. D 97, 114024 (2018). 
[13] A. J. Arifi, H. Nagahiro, and A. Hosaka, Three-body decay of $\Lambda_{c}^{*}(2595)$ and $\Lambda_{c}^{*}(2625)$ with the inclusion of a direct two-pion coupling, Phys. Rev. D 98, 114007 (2018).

[14] T. Aaltonen et al. (CDF Collaboration), Measurements of the properties of $\Lambda_{c}(2595), \Lambda_{c}(2625), \Sigma_{c}(2455)$, and $\Sigma_{c}(2520)$ baryons, Phys. Rev. D 84, 012003 (2011).

[15] R. Aaij et al. (LHCb Collaboration), Observation of Excited $\Lambda_{b}^{0}$ Baryons, Phys. Rev. Lett. 109, 172003 (2012).

[16] T. A. Aaltonen et al. (CDF Collaboration), Evidence for a bottom baryon resonance $\Lambda_{b}^{* 0}$ in CDF data, Phys. Rev. D 88, 071101 (2013).

[17] P. L. Cho, Strong and electromagnetic decays of two new $\Lambda_{c}^{*}$ baryons, Phys. Rev. D 50, 3295 (1994).

[18] N. Jiang, X. L. Chen, and S. L. Zhu, Electromagnetic decays of the charmed and bottom baryons in chiral perturbation theory, Phys. Rev. D 92, 054017 (2015).

[19] H. Y. Cheng and C. K. Chua, Strong decays of charmed Baryons in heavy hadron chiral perturbation theory: An update, Phys. Rev. D 92, 074014 (2015).

[20] M. A. Ivanov, J. G. Korner, V. E. Lyubovitskij, and A. G. Rusetsky, Strong and radiative decays of heavy flavored baryons, Phys. Rev. D 60, 094002 (1999).

[21] T. Yoshida, E. Hiyama, A. Hosaka, M. Oka, and K. Sadato, Spectrum of heavy baryons in the quark model, Phys. Rev. D 92, 114029 (2015).

[22] A. Hosaka, E. Hiyama, S. H. Kim, H.-C. Kim, H. Nagahiro, H. Noumi, M. Oka, K. Shirotori, T. Yoshida, and S. Yasui, Production and decay of charmed baryons, Nucl. Phys. A954, 341 (2016).

[23] H. Nagahiro, S. Yasui, A. Hosaka, M. Oka, and H. Noumi, Structure of charmed baryons studied by pionic decays, Phys. Rev. D 95, 014023 (2017).

[24] A. J. Arifi, H. Nagahiro, and A. Hosaka, Three-body decay of $\Lambda_{c}^{*}(2595)$ and $\Lambda_{c}^{*}(2625)$ with consideration of $\Sigma_{c}(2455) \pi$ and $\Sigma_{c}^{*}(2520) \pi$ in intermediate States, Phys. Rev. D 95, 114018 (2017).

[25] K. L. Wang, Y. X. Yao, X. H. Zhong, and Q. Zhao, Strong and radiative decays of the low-lying $S$ - and $P$-wave singly heavy baryons, Phys. Rev. D 96, 116016 (2017).

[26] K. Gandhi, Z. Shah, and A. K. Rai, Decay properties of singly charmed baryons, Eur. Phys. J. Plus 133, 512 (2018).
[27] H. X. Chen, W. Chen, Q. Mao, A. Hosaka, X. Liu, and S. L. Zhu, $P$-wave charmed baryons from QCD sum rules, Phys. Rev. D 91, 054034 (2015).

[28] Q. Mao, H. X. Chen, W. Chen, A. Hosaka, X. Liu, and S. L. Zhu, QCD sum rule calculation for $P$-wave bottom baryons, Phys. Rev. D 92, 114007 (2015).

[29] H. X. Chen, Q. Mao, W. Chen, A. Hosaka, X. Liu, and S. L. Zhu, Decay properties of $P$-wave charmed baryons from light-cone QCD sum rules, Phys. Rev. D 95, 094008 (2017).

[30] K. Thakkar, Z. Shah, A. K. Rai, and P. C. Vinodkumar, Excited state mass spectra and Regge trajectories of bottom Baryons, Nucl. Phys. A965, 57 (2017).

[31] H. Bahtiyar, K. U. Can, G. Erkol, M. Oka, and T. T. Takahashi, $\Xi_{c} \gamma \rightarrow \Xi_{c}^{\prime}$ transition in lattice QCD, Phys. Lett. B 772, 121 (2017).

[32] H. Bahtiyar, K. U. Can, G. Erkol, M. Oka, and T. T. Takahashi, Radiative transitions of doubly charmed baryons in lattice QCD, Phys. Rev. D 98, 114505 (2018).

[33] F. K. Guo, C. Hanhart, U. G. Meißner, Q. Wang, Q. Zhao, and B. S. Zou, Hadronic molecules, Rev. Mod. Phys. 90, 015004 (2018).

[34] J. X. Lu, Y. Zhou, H. X. Chen, J. J. Xie, and L. S. Geng, Dynamically generated $J^{P}=1 / 2^{-}\left(3 / 2^{-}\right)$singly charmed and bottom heavy baryons, Phys. Rev. D 92, 014036 (2015).

[35] H. X. Chen, W. Chen, X. Liu, Y. R. Liu, and S. L. Zhu, A review of the open charm and open bottom systems, Rep. Prog. Phys. 80, 076201 (2017).

[36] M. Tanabashi et al. (Particle Data Group), Review of particle physics, Phys. Rev. D 98, 030001 (2018).

[37] B. Chen, K. W. Wei, X. Liu, and A. Zhang, Role of newly discovered $\Xi_{b}(6227)^{-}$for constructing excited bottom baryon family, Phys. Rev. D 98, 031502 (2018).

[38] R. Aaij et al. (LHCb Collaboration), Observation of a New $\Xi_{b}^{-}$Resonance, Phys. Rev. Lett. 121, 072002 (2018).

[39] Y. Huang, C. j. Xiao, L. S. Geng, and J. He, Strong decays of the $\Xi_{b}(6227)$ as a $\Sigma_{b} \bar{K}$ molecule, Phys. Rev. D 99, 014008 (2019).

[40] V. Dmitrašinović, H.-X. Chen, and A. Hosaka (private communication). 